\title{
Importance of Fungicide Seed Treatment and Environment on Seedling Diseases of Cotton
}

C. S. Rothrock, S. A. Winters, and P. K. Miller, Department of Plant Pathology, University of Arkansas, Fayetteville 72701; E. Gbur, Agricultural Statistics, University of Arkansas, Fayetteville 72701; L. M. Verhalen and B. E. Greenhagen, Department of Plant and Soil Sciences, Oklahoma State University, Stillwater 74078; T. S. Isakeit, Department of Plant Pathology and Microbiology, Texas A\&M University, College Station 77843; W. E. Batson, Jr., Department of Entomology and Plant Pathology, Mississippi State University, Mississippi State 39762; F. M. Bourland, Department of Crop, Soil, and Environmental Sciences, University of Arkansas, Northeast Research and Extension Center, Keiser 72351; P. D. Colyer, Red River Research Station, Louisiana State University AgCenter, Bossier City 71113; T. A. Wheeler and H. W. Kaufman, Texas AgriLife Research and Extension Center, Lubbock 79403; G. L. Sciumbato and P. M. Thaxton, Mississippi State University, Delta Research and Extension Center, Stoneville 38776; K. S. Lawrence and W. S. Gazaway, Department of Entomology and Plant Pathology, Auburn University, Auburn 36849; A. Y. Chambers and M. A. Newman, Department of Entomology and Plant Pathology, University of Tennessee, West Tennessee Research and Education Center, Jackson 38301; T. L. Kirkpatrick and J. D. Barham, Department of Plant Pathology, University of Arkansas, Southwest Research and Extension Center, Hope 71801; P. M. Phipps and F. M. Shokes, Virginia Polytechnic Institute and State University, Tidewater Agriculture Research and Extension Center, Suffolk 23437; L. J. Littlefield, Department of Entomology and Plant Pathology, Oklahoma State University, Stillwater 74078; G. B. Padgett, Macon Ridge Research Station, Louisiana State University AgCenter, Winnsboro, 71295; R. B. Hutmacher, University of California, Davis, Shafter Research and Extension Center, Shafter 93263; R. M. Davis, Department of Plant Pathology, University of California, Davis 95616; R. C. Kemerait and D. R. Sumner, Department of Plant Pathology, University of Georgia, Tifton 31794; K. W. Seebold, Jr., Department of Plant Pathology, University of Kentucky, Lexington 40546; J. D. Mueller, Clemson University, Edisto Research and Education Center, Blackville 29817; and R. H. Garber, USDA-ARS, Shafter 93263

\begin{abstract}
Rothrock, C. S., Winters, S. A., Miller, P. K., Gbur, E., Verhalen, L. M., Greenhagen, B. E., Isakeit, T. S., Batson, W. E., Jr., Bourland, F. M., Colyer, P. D., Wheeler, T. A., Kaufman, H. W., Sciumbato, G. L., Thaxton, P. M., Lawrence, K. S., Gazaway, W. S., Chambers, A. Y., Newman, M. A., Kirkpatrick, T. L., Barham, J. D., Phipps, P. M., Shokes, F. M., Littlefield, L. J., Padgett, G. B., Hutmacher, R. B., Davis, R. M., Kemerait, R. C., Sumner, D. R., Seebold, K. W., Jr., Mueller, J. D., and Garber, R. H. 2012. Importance of fungicide seed treatment and environment on seedling diseases of cotton. Plant Dis. 96:1805-1817.

The importance of fungicide seed treatments on cotton was examined using a series of standardized fungicide trials from 1993 to 2004. Fungicide seed treatments increased stands over those from seed not treated with fungicides in 119 of 211 trials. Metalaxyl increased stands compared to nontreated seed in 40 of 119 trials having significant fungicide responses, demonstrating the importance of Pythium spp. on stand establishment. Similarly, PCNB seed treatment increased stands compared to nontreated seed for 44 of 119 trials with a significant response, indicating the importance of Rhizoctonia solani in stand losses. Benefits from the use of newer seed treatment chemistries,

historic standard seed treatment, carboxin + PCNB + metalaxyl. Little to no stand improvement was found when minimal soil temperatures averaged $25^{\circ} \mathrm{C}$ the first 3 days after planting. Stand losses due to seedling pathogens increased dramatically as minimal soil temperatures decreased to $12^{\circ} \mathrm{C}$ and rainfall increased. The importance of Pythium increased dramatically as minimal soil temperature decreased and rainfall increased, while the importance of $R$. solani was not affected greatly by planting environment. These multi-year data support the widespread use of seed treatment fungicides for the control of the seedling disease complex on cotton.
\end{abstract} azoxystrobin and triazoles, were demonstrated by comparison with a
Seedling diseases of cotton (Gossypium hirsutum L.) collectively refer to a group of diseases that affect the germination of cottonseed and emergence, survival, and development of seedlings. Cotton seedling diseases are a major factor affecting cotton production worldwide $(13,20,36,38)$. Cotton disease loss estimates for the United States from 1952 to 2009 averaged 2.8\%, with loss estimates for seedling diseases accounting for $23 \%$ of the total estimated losses in lint production over these years (Disease data-

Corresponding author: C. S. Rothrock, E-mail: Rothrock@uark.edu

Accepted for publication 2 July 2012.

http://dx.doi.org/10.1094/PDIS-01-12-0031-SR

(c) 2012 The American Phytopathological Society base, http://www.cotton.org/tech/pest/seedling/index.cfm). In the field, the most obvious symptoms of cotton seedling diseases are skips in the planting row resulting from rotted seed or dead seedlings. In addition to stand losses, seedling diseases may delay crop growth, resulting in additional management problems, such as timing of pesticide applications and harvest. In severe disease situations, replanting may be required.

The most important pathogens in the cotton seedling disease complex are Rhizoctonia solani Kühn, teleomorph Thanatephorus cucumeris (A.B. Frank) Donk, Pythium spp., Fusarium spp., and Thielaviopsis basicola (Berk. \& Broome) Ferraris (syn. Chalara elegans Nag Raj \& Kendrick) $(10,11,13,17,24,36,59,60)$. These pathogens act either alone or in combination to cause a range of symptoms on roots and hypocotyls $(13,17,24,59)$.

Pythium spp. can cause severe stand loss in cotton $(14,17$, 21,24,38). Johnson et al. (24) found that Pythium spp. were the 
most frequently isolated pathogen from diseased cotton seedlings. Many species of Pythium contribute to the cotton seedling disease complex $(14,21,24,25)$. The most common symptoms associated with Pythium spp. are seed rot and preemergence damping-off $(3,21)$. In Tennessee, a significant negative correlation was obtained between percentage of seedlings from which Pythium spp. was isolated and percent emergence (27).

Ranney (40) and Davis (11) in Mississippi and Ray and McLaughlin in Oklahoma (41) reported $R$. solani to be the most important pathogen associated with diseased cotton seedlings. $R$. solani has been consistently associated with all stages of seedling disease. Fulton and Bollenbacher (17) and Ogle et al. (38) reported preemergence death of cotton due to $R$. solani, but damage caused by $R$. solani is most often associated with postemergence damping-off $(47,60)$.

Fusarium spp. are often the most frequently isolated fungal genus from cotton roots $(8,17,24,27,36,41,59)$. A number Fusarium spp. are isolated from cotton seedlings $(1,8,39,59)$. Results from pathogenicity tests of Fusarium spp. indicated that most species are of minor significance in the etiology of cotton seedling disease $(17,24,36)$. Johnson and Doyle (27) found a negative correlation between disease severity and isolation frequency of Fusarium spp., while Roy and Bourland (59) found a positive correlation between incidence of Fusarium oxysporum Schlechtend.:Fr. and disease severity on seedlings. Pathogenicity for isolates of several species, most frequently $F$. oxysporum and $F$. solani (Mart.) Sacc., have been demonstrated $(1,8,9,36,59)$.

Black root rot caused by $T$. basicola is considered part of the cotton seedling disease complex $(2,27,33,59)$. Symptoms associated with $T$. basicola include blackening of the roots and the belowground portion of the hypocotyls and stunting of cotton seedlings $(2,36,44)$. In a Mississippi survey, T. basicola was detected in 18 of 36 locations and observed on $100 \%$ of cotton seedlings for four locations (59). T. basicola was found in over $70 \%$ of cotton fields surveyed in Arkansas and Texas $(49,66)$. For surveys in Mississippi, incidence of T. basicola was negatively correlated with cotton stand and positively correlated with root and hypocotyl disease indices (59).

Growers often plant cotton early to maximize the growing season and to avoid losses from insect damage or rain late in the sea- son. Soil temperatures, as well as soil moisture, during the first few weeks after planting are important in stand establishment, because of effects on both the host and the pathogens $(26,29,37,42)$. Cotton seedling disease control relies on avoidance, delaying planting until soil temperatures become favorable for cotton germination and growth with a favorable weather forecast, along with planting high-quality seed, proper planting depth, and planting on a wellprepared raised seedbed in well-drained soils $(15,20,29,37,60)$. In addition, cottonseed in the United States is universally treated with combinations of fungicides prior to sale to protect the crop from a range of seedling disease pathogens, but also as a result of centralized seed production and sales due to the delinting process and the use of transgenic cultivars $(12,20,37)$. The National Cottonseed Treatment Program has evaluated cotton seedling survival for commercial fungicide seed treatment combinations over diverse environmental conditions as part of the activities of the Cotton Disease Council. Since 1993, a historic standard fungicide treatment, carboxin + PCNB + metalaxyl, and a nontreated control were included to assess improvements in the efficacy of seed treatment fungicides and document the importance of seedling diseases. In addition, selective fungicide treatments were used to aid in determining the importance of Pythium spp. or $R$. solani. The objectives of this report were to examine the importance of seedling diseases on cotton, the role of specific pathogens, and the role of environment on stand establishment on cotton. Annual results of these trials have been published previously $(45,46,48,50-58)$.

\section{Materials and Methods}

Fungicide treatment. Acid-delinted neutralized seed of Gossypium hirsutum L. were provided by Stoneville Pedigreed Seed Company, Stoneville, MS, in 1993 and 1994, and Delta and Pine Land Company, Scott, MS, from 1995 to 2004. Cultivars were Stoneville 453 (PVP 88001730) in 1993 and 1994, Deltapine 50 (PVP 8400154) in 1995 to 2000, Sure-Grow 747 (PVP 9800118) in 2001, and Deltapine DP 451 B/RR (PVP 9900294) in 2002 to 2004, except for locations in Oklahoma and the Lubbock and College Station locations in Texas in 1995 to 2004, where the cultivars were Paymaster HS26 (PVP 8600087) in 1995 to 2000 and Paymaster PM 2326 RR (PVP 9900191) in 2001 to 2004. A rotat-

Table 1. Active ingredients and products included in analyses of the 1993-2004 National Cottonseed Treatment Program

\begin{tabular}{|c|c|c|}
\hline Common name & Product & Active ingredient \\
\hline Azoxystrobin & $\begin{array}{l}\text { Protégé FL or } 70 \mathrm{WP} \\
\text { Dynasty CST } \\
\text { Azoxystrobin } 100 \mathrm{FS}\end{array}$ & Methyl (E)-2-\{2-[6-(2-cyanophenoxy)pyrimidin-4-yloxy]phenyl $\}-3$-methoxyacrylate \\
\hline Captan & $\begin{array}{l}\text { Captan } 4000 \\
\text { Nu-Gro }\end{array}$ & $N$-(trichloromethylthio)-4-cyclohexene-1,2-dicarboximide \\
\hline Carboxin & $\begin{array}{l}\text { Vitavax-PCNB } \\
\text { Vitavax } 200\end{array}$ & 5,6-dihydro-2-methyl- $N$-phenyl-1,4-oxathiin-3-carboxamide \\
\hline Chloroneb & Nu-Flow AD, D, or ND & 1,4-dichloro-2,5-dimethoxy-benzene \\
\hline Fludioxonil & $\begin{array}{l}\text { Maxim 4FS } \\
\text { Dynasty CST }\end{array}$ & 4-(2,2-difluoro-1,3-benzodioxol-4-yl)-1 $H$-pyrrole-3-carbonitrile, \\
\hline Mefenoxam & $\begin{array}{l}\text { Apron XL } \\
\text { Dynasty CST }\end{array}$ & $(R)$-2-[(2,6-dimethylphenyl)-methoxyacetylamino]-propionic acid methyl ester \\
\hline Metalaxyl & $\begin{array}{l}\text { Allegiance FL or LS } \\
\text { Apron FL, 3FS, or TL } \\
\text { Nu-Flow AD }\end{array}$ & $N$-(2,6-dimethylphenyl)- $N$-(methoxyacetyl) alanine methyl ester \\
\hline Myclobutanil & $\begin{array}{l}\text { Nu-Flow M } \\
\text { Systane WP }\end{array}$ & (alpha- $n$-butyl-alpha-(4-chlorophenyl)- $1 H$-1,2,4-triazole-1-propanenitrile \\
\hline PCNB & $\begin{array}{l}\text { RTU-PCNB } \\
\text { Vitavax } 200 \\
\text { Vitavax- PCNB }\end{array}$ & Pentachloronitrobenzene \\
\hline ТСМТВ & $\begin{array}{l}\text { Argent } 30 \\
\text { Ascend } \\
\text { Nu-Flow T or ND } \\
\text { Nusan } 30 \\
\text { Busan 30AU }\end{array}$ & 2-(thiocyanomethylthio)benzothiazole \\
\hline Thiram & $\begin{array}{l}\text { Thiram } 42-\mathrm{S} \text { or } 75 \mathrm{WDG} \\
\text { RTU-Baytan-Thiram }\end{array}$ & Tetramethylthiuram disulfide \\
\hline Triadimenol & $\begin{array}{l}\text { Baytan } 30 \\
\text { RTU-Baytan-Thiram }\end{array}$ & Beta-(4-chlorophenoxy)-alpha-(1,1-dimethylethyl)-1H-1,2,4-triazole-1-ethanol \\
\hline
\end{tabular}


ing drum seed treater was used from 1993 to 2002, and a Hege 11 liquid seed treater (Hege Maschinen $\mathrm{GmbH}$, Waldenburg, Germany) was used in 2003 and 2004. Fungicides were applied as $2 \%$ liquid in water (vol/wt) from 1993 to $2000,2.5 \%$ in 2001, $2.6 \%$ in 2002, and $2.5 \%$ in 2003 (PCNB was applied at $2.6 \% \mathrm{vol} / \mathrm{wt}$ ). Colorant dye was added to seed starting in 1999; Pro-Ized or DayGlo EPX seed colorant dye was added in 1999 or 2000 to 2002, respectively (Gustafson LLC), and Color Coat Red (Syngenta Inc.) in 2003. In 2004, fungicide treatments were mixed with $\mathrm{CaCO}_{3}$ (437 g/100 kg seed), polymer (Secure $65 \mathrm{ml} / 100 \mathrm{~kg}$ seed, Syngenta Inc.), and dye (Color Coat Red $65 \mathrm{ml} / 100 \mathrm{~kg}$ seed, Syngenta Inc.) in water at a rate of $2.5 \%$ water to seed weight (wt/wt) (PCNB was applied at $2.62 \% \mathrm{wt} / \mathrm{wt}$ ). Seed not receiving a fungicide treatment were treated in the same manner as fungicide-treated seed. When two or more fungicides were applied, the fungicides were mixed and applied in a single application, except in 1993 and 1994 when some fungicides were added sequentially. The common seed treatments across all years were no fungicide seed treatment, metalaxyl, PCNB (carboxin + PCNB in 1993), and carboxin + PCNB + metalaxyl. All other fungicide combinations were nominated by industry representatives and ranged from 12 to 16 fungicide treatment combinations for the 1993 to 2004 evaluations (Table 1).

Field experiments. Field experiments were conducted across the Cotton Belt as part of cooperative efforts of members of the Cotton Disease Council to evaluate the efficacy of seed treatment combinations used by the cottonseed industry and experimental compounds (Table 2). The number of trials where data were recorded ranged from 15 trials in 2001 to 20 trials in 1996 for a total of 211 trials. Each trial utilized a randomized complete block experimental design, with the number of replications ranging from
4 to 10 . For each trial, stand counts used in the analyses were taken at approximately 31 days after planting depending on the location and year. The mean date for taking stand counts from trials for each year varied from 29 to 35 days after planting over the 12 years. Seedlings were dug from the plots not receiving a fungicide treatment by arbitrarily selecting $0.3-\mathrm{m}$ sections of row and digging up approximately 50 seedlings per site, 100 seedlings per site from 2001 to 2004. A composite soil sample from the top $15 \mathrm{~cm}$ of soil was also obtained for each site starting in 1994. Soil and seedlings were placed in insulated packages with refrigerated cool packs and mailed overnight to the University of Arkansas for processing. Soil temperature and moisture was monitored by burying a temperature sensor and a Watermark soil moisture sensor connected to a data logger (Spectrum Technologies, Inc., Plainfield, IL) $10 \mathrm{~cm}$ deep at planting starting in 1999. Rainfall data were obtained from the test site or the nearest NOAA site for each trial (http://www.ncdc.noaa.gov/oa/ncdc.html).

Seedlings were evaluated for growth by recording the number of nodes from five arbitrarily selected seedlings, and then the aboveground portions of all seedlings were removed and discarded. Seedlings for a location were then rinsed for at least $20 \mathrm{~min}$ in running tap water and rated for disease symptoms. The hypocotyl disease severity index was $1=$ no symptoms, $2=$ few pinpoint lesions or diffuse discolored areas, $3=$ distinct necrotic lesion, $4=$ girdling lesion, and $5=$ seedling dead. The root disease index was $1=$ no symptoms, $2=1-10 \%$ of the root system discolored, $3=$ $11-25 \%$ of the root system discolored, $4=26-50 \%$ of the root system discolored, and $5>50 \%$ of the root system discolored. Seedlings were surface disinfested by immersion for $1.5 \mathrm{~min}$ in $0.5 \% \mathrm{NaClO}$, blotted dry in a paper towel, and plated on water agar $(0.8 \%)$ amended with 10 and $250 \mathrm{mg}$ of the antibiotics rifampicin

Table 2. Fungicide seed treatment trials conducted as part of the National Cottonseed Treatment Program from 1993 to 2004

\begin{tabular}{|c|c|c|c|c|c|c|c|}
\hline Year & Cooperator & Location & Site & Rep. & $\begin{array}{l}\text { Row length } \\
\text { (m) }\end{array}$ & Seed/m & Soil type \\
\hline $1993-2004$ & Gazaway, Lawrence & Auburn, AL & $\mathrm{AL}$ & $4-6$ & $3-8$ & $16-23$ & $\begin{array}{l}\text { Decatur silt loam or Lucedale fine } \\
\text { sandy loam }\end{array}$ \\
\hline $1993-2004$ & Kirkpatrick, Barham & Hope, AR & AR1 & 5 & $8-18$ & $12-20$ & Various \\
\hline 1993-2004 & Rothrock, Bourland & Keiser, AR & AR2 & $4-9$ & $6-24$ & $12-25$ & Sharkey silty clay \\
\hline 1994-2004 & Rothrock & Clarkedale, AR & AR3 & $6-8$ & 15 & 16 & Dundee silt loam \\
\hline $1993-2004$ & $\begin{array}{l}\text { Hutmacher, Davis, } \\
\text { Garber }\end{array}$ & Shafter, CA & $\mathrm{CA}$ & $5-8$ & $6-8$ & $16-20$ & $\ldots^{\mathrm{z}}$ \\
\hline 1994-1998 & Shokes & Quincy, FL & FL & $5-6$ & $7-8$ & $14-23$ & Norfolk loamy fine sand \\
\hline 1997 & Shokes & Marianna, FL & FL2 & 6 & 7 & 14 & Chipola fine sandy loam \\
\hline $1993-2004$ & $\begin{array}{l}\text { Sumner, Seebold, } \\
\text { Kemerait }\end{array}$ & Tifton, GA & GA & $4-5$ & $6-12$ & $8-16$ & Tifton loamy sand \\
\hline 1993-2004 & Colyer & Bossier City, LA & LA1a & $5-6$ & 8 & 13 & Norwood very fine sandy loam \\
\hline 1998 & Colyer & St. Joseph, LA & LA1b & 4 & 8 & 13 & Bruin and Commerce sandy loam \\
\hline 1993-1998 & Lawrence & Monroe, LA & LA2 & 5 & 12 & $17-20$ & Rilla-Hebert silt loam \\
\hline $1999-2004$ & Padgett & Winnsboro, LA & LA2 & $4-5$ & $6-8$ & $13-15$ & Gigger silt loam \\
\hline 1993-2004 & Batson & Mississippi State, MS & MS1 & $4-5$ & $12-49$ & $10-25$ & Marietta loam or Brooksville silty loam \\
\hline 1993-2004 & Sciumbato & Stoneville, MS & MS2 & $4-5$ & $12-14$ & $16-20$ & Bosket fine sandy loam \\
\hline $1993-2004$ & $\begin{array}{l}\text { Verhalen, Littlefield, } \\
\text { Greenhagan (VLG) }\end{array}$ & Tipton, OK & OK1 & 4 & $6-7$ & $15-16$ & Tipton silt loam \\
\hline 1993-2004 & VLG & Altus, OK & OK2 & 4 & $6-7$ & $15-16$ & Hollister clay loam \\
\hline 1993-2004 & VLG & Perkins, OK & OK3 & 4 & $6-7$ & $15-16$ & Teller loam \\
\hline $1993-1996$ & Mueller & Edisto, SC & $\mathrm{SC}$ & $4-5$ & $8-12$ & 13 & $\ldots$ \\
\hline 1993-2004 & Chambers, Newman & Jackson, TN & $\mathrm{TN}$ & $8-10$ & $5-8$ & $13-22$ & Dexter loam \\
\hline 1993-2001 & Thaxton, Isakeit & College Station, TX & TX1 & $4-8$ & $6-12$ & $10-16$ & Lufkin fine sandy loam \\
\hline $1993-2000$ & Kaufman, Wheeler & Lubbock, TX & TX2 & $4-6$ & $4-11$ & $16-20$ & Acuff sandy clay loam \\
\hline 2001-2004 & Kaufman, Wheeler & Halfway, TX & TX2 & 4 & 11 & 16 & Pullman clay loam \\
\hline $1995-1998$ & Isakeit & Weslaco, TX & TX3 & 4 & 6 & 16 & $\begin{array}{l}\text { Raymondville clay loam or Hidalgo } \\
\text { sandy clay loam }\end{array}$ \\
\hline 2002-2003 & Isakeit & Victoria, TX & TX3 & 4 & 5 & 19 & Lake Charles clay \\
\hline 1996-1997 & Isakeit & Edroy, TX & TX3b & 4 & 6 & 16 & Victory clay \\
\hline 2002 & Isakeit & Brazos, TX & TX4 & 4 & 5 & 19 & $\ldots$ \\
\hline 2003-2004 & Isakeit & Beaumont, TX & TX4 & 4 & $5-6$ & 16 & Morey silt loam \\
\hline 1999-2001 & Isakeit & Burleson Co., TX & TX3 & 4 & $5-6$ & $16-19$ & Westwood silt loam \\
\hline 2002-2004 & & & TX5 & 4 & 5 & $18-19$ & Miller-Norwood Pledger clay loam \\
\hline $1998-2004$ & P. M. Phipps & Suffolk, VA & VA & 4 & 18 & $10-13$ & $\begin{array}{l}\text { Kenansville loamy sand or Nansemond } \\
\text { fine sandy loam }\end{array}$ \\
\hline
\end{tabular}

\footnotetext{
${ }^{\mathrm{z}}$ Soil type not reported.
} 
and ampicillin, respectively, and the miticide fenpropathrin $(0.14$ mg a.i./liter, Danitol 2.4 EC, Valent Chemical Co.). Resulting colonies were transferred to potato dextrose agar (PDA) and identified to genus. After 5 days, the seedlings on water agar were transferred to the Thielaviopsis selective medium TB-CEN (62), which was modified by adding penicillin $\mathrm{G}(60 \mathrm{mg} / \mathrm{liter})$, to determine isolation frequency of $T$. basicola for the same seedlings as evaluated for other pathogens. A separate evaluation of isolation frequency for Pythium was conducted using an additional set of seedlings for sites, up to 50 seedlings, starting in 2001. Isolation was done by plating seedlings, following a 20-min water rinse, on the selective medium $\mathrm{P}_{5}$ ARP (23). This allowed a different assessment of incidence of Pythium spp. from the surface disinfestation technique with $\mathrm{NaOCl}$ and isolation on water agar.

Soil samples were assayed for populations of Rhizoctonia spp. by using the multiple-pellet soil method (19) on a modified Ko and

Table 3. Plant stands for fungicide seed treatment combinations used for analyses from trials with a significant treatment response from the National Cottonseed Treatment Program from 1993 to $1998^{t}$

\begin{tabular}{|c|c|c|c|c|c|c|c|c|c|c|c|c|c|}
\hline $\begin{array}{l}\text { Year } \\
\text { Treatment }\end{array}$ & $\begin{array}{l}\text { Rate } \\
(\text { g a.i./100 kg seed })^{v}\end{array}$ & & & & & & lant $s$ & and $(\%$ & & & & & \\
\hline 1993 & & $\mathrm{AL}$ & AR1 & LA2 & MS1 & MS2 & OK1 & $\mathrm{TN}$ & TX1a ${ }^{w}$ & TX1b $1 b^{w}$ & & & \\
\hline Triadimenol+metalaxyl+thiram & $20.7+15.2+62.5$ & 34 & 49 & 62 & 74 & 26 & 74 & 33 & 48 & 85 & & & \\
\hline Metalaxyl+myclobutanil+captan & $16.2+43.5+94.5$ & 32 & 44 & 48 & 72 & 32 & 85 & 42 & 47 & 84 & & & \\
\hline Metalaxyl+myclobutanil+TCMTB & $16.2+43.5+45.7$ & 33 & 46 & 24 & 74 & 36 & 81 & 40 & 45 & 84 & & & \\
\hline Triadimenol+metalaxyl+thiram & $10.4+15.2+62.5$ & 35 & 42 & 44 & 70 & 18 & 76 & 27 & 46 & 88 & & & \\
\hline Metalaxyl+myclobutanil & $16.2+43.5$ & 24 & 48 & 24 & 67 & 38 & 79 & 49 & 46 & 87 & & & \\
\hline Chloroneb-metalaxyl+myclobutanil & $130.3+5.2+43.5$ & 29 & 46 & 29 & 69 & 20 & 82 & 43 & 41 & 80 & & & \\
\hline Carboxin-PCNB+metalaxyl & $77.1+77.1+15.2$ & 39 & 44 & 40 & 64 & 18 & 76 & 36 & 48 & 86 & & & \\
\hline Carboxin-PCNB & $77.1+77.1$ & 28 & 34 & 24 & 59 & 28 & 74 & 24 & 25 & 68 & & & \\
\hline Metalaxyl & 15.2 & 34 & 40 & 32 & 66 & 6 & 73 & 24 & 47 & 80 & & & \\
\hline None & & 19 & 31 & 33 & 62 & 10 & 70 & 16 & 26 & 60 & & & \\
\hline Location average & & 32 & 43 & 40 & 67 & 23 & 77 & 34 & 42 & 81 & & & \\
\hline $\operatorname{LSD}(P=0.05)^{\mathrm{x}}$ & & 11.1 & 7.0 & 13.7 & 10.2 & 17.2 & 8.6 & 18.0 & 11.1 & 8.4 & & & \\
\hline 1994 & & AR1 & AR2 & GA & LA1 & LA2 & MS1 & MS2 & $\mathrm{TN}$ & TX1 & TX2 & & \\
\hline Triadimenol+metalaxyl+thiram & $20.7+15.2+62.5$ & 66 & 61 & 43 & 50 & 97 & 76 & 50 & 83 & 73 & 26 & & \\
\hline Triadimenol+metalaxyl+thiram & $10.4+15.2+62.5$ & 64 & 66 & 54 & 40 & 92 & 74 & 42 & 82 & 69 & 27 & & \\
\hline Captan+metalaxyl+myclobutanil & $78.8+16.2+43.5$ & 65 & 63 & 49 & 44 & 94 & 76 & 42 & 79 & 68 & 34 & & \\
\hline $\begin{array}{l}\text { Chloroneb-metalaxyl+myclobutanil } \\
\text { +Trichoderma harzianum }\end{array}$ & $130.3+15.2+43.5+3.6$ & 65 & 64 & 39 & 41 & 87 & 69 & 45 & 77 & 67 & 24 & & \\
\hline Captan+metalaxyl+myclobutanil & $78.8+16.2+21.8$ & 62 & 67 & 41 & 37 & 88 & 74 & 38 & 78 & 70 & 23 & & \\
\hline Carboxin-PCNB+metalaxyl & $77.1+77.1+15.2$ & 64 & 61 & 31 & 40 & 81 & 71 & 36 & 74 & 63 & 24 & & \\
\hline PCNB & 252.8 & 65 & 47 & 29 & 39 & 70 & 74 & 46 & 64 & 60 & 17 & & \\
\hline Metalaxyl & 30.4 & 55 & 73 & 19 & 19 & 84 & 71 & 38 & 77 & 62 & 33 & & \\
\hline None & & 51 & 50 & 29 & 21 & 66 & 69 & 33 & 64 & 52 & 15 & & \\
\hline Location average & & 62 & 63 & 38 & 37 & 84 & 73 & 42 & 75 & 65 & 26 & & \\
\hline $\operatorname{LSD}(P=0.05)$ & & 6.9 & 13.4 & 20.0 & 10.2 & 16.1 & 5.2 & 8.1 & 7.0 & 6.8 & 10.6 & & \\
\hline 1995 & & AR1 & AR2 & AR3 & $\mathrm{CA}$ & GA & LA1 & OK1 & $\mathrm{OK} 3$ & $\mathrm{TN}$ & TX1 & TX2 & \\
\hline TCMTB+triadimenol+metalaxyl & $101.6+10.4+20.2$ & 58 & 44 & 63 & 83 & 70 & 81 & 82 & 68 & 67 & 83 & 59 & \\
\hline TCMTB+myclobutanil+metalaxyl & $40.6+21.8+16.2$ & 58 & 42 & 52 & 81 & 68 & 78 & 78 & 70 & 69 & 79 & 62 & \\
\hline Triadimenol+metalaxyl+thiram & $20.7+15.2+62.5$ & 61 & 40 & 61 & 80 & 73 & 76 & 74 & 65 & 72 & 82 & 51 & \\
\hline Triadimenol+metalaxyl+thiram & $10.4+15.2+62.5$ & 46 & 38 & 58 & 76 & 66 & 75 & 77 & 63 & 71 & 84 & 55 & \\
\hline Triadimenol-thiram+metalaxyl & $10.6+32.0+15.2$ & 64 & 40 & 59 & 76 & 70 & 81 & 71 & 62 & 66 & 82 & 47 & \\
\hline Thiram+myclobutanil+metalaxyl & $63.2+30.5+16.2$ & 64 & 43 & 54 & 78 & 67 & 72 & 71 & 61 & 70 & 83 & 53 & \\
\hline Thiram+myclobutanil+metalaxyl & $63.2+21.8+16.2$ & 54 & 42 & 57 & 80 & 70 & 74 & 74 & 66 & 67 & 80 & 56 & \\
\hline TCMTB+myclobutanil+metalaxyl & $40.6+43.5+16.2$ & 57 & 34 & 60 & 80 & 71 & 72 & 73 & 68 & 69 & 85 & 53 & \\
\hline $\begin{array}{l}\text { Chloroneb-metalaxyl+myclobutanil } \\
\text { +TCMTB }\end{array}$ & $130.3+15.2+43.5+40.6$ & 61 & 42 & 54 & 84 & 64 & 72 & 75 & 58 & 69 & 83 & 55 & \\
\hline TCMTB+myclobutanil+metalaxyl & $50.8+30.5+16.2$ & 63 & 36 & 50 & 72 & 66 & 74 & 70 & 64 & 70 & 83 & 61 & \\
\hline $\begin{array}{l}\text { Thiram-triadimenol+PCNB+ } \\
\text { metalaxyl }\end{array}$ & $21.3+7.1+52.3+15.2$ & 62 & 35 & 52 & 82 & 76 & 72 & 74 & 58 & 72 & 78 & 56 & \\
\hline Carboxin-PCNB+metalaxyl & $77.1+77.1+15.2$ & 58 & 40 & 52 & 82 & 51 & 73 & 76 & 64 & 66 & 80 & 53 & \\
\hline $\mathrm{PCNB}$ & 252.8 & 59 & 12 & 55 & 69 & 54 & 71 & 74 & 49 & 50 & 71 & 42 & \\
\hline Metalaxyl & 30.4 & 47 & 46 & 40 & 76 & 65 & 59 & 66 & 51 & 63 & 67 & 44 & \\
\hline None & & 41 & 15 & 42 & 76 & 61 & 61 & 58 & 32 & 41 & 52 & 34 & \\
\hline Location average & & 57 & 37 & 55 & 78 & 68 & 73 & 74 & 61 & 65 & 79 & 54 & \\
\hline $\operatorname{LSD}(P=0.05)$ & & 4.9 & 10.7 & 10.6 & 9.9 & 14.1 & 10.2 & 10.5 & 7.7 & 8.6 & 5.6 & 12.2 & \\
\hline 1996 & & AR2 & AR3 & CA & FL & GA & LA1 & OK1 & $\mathrm{OK} 2$ & OK3 & $\mathrm{TN}$ & TX1 & $\mathrm{TX} 2$ \\
\hline Triadimenol+FB23 $3^{\mathrm{y}}+$ metalaxyl & $10.4+\mathrm{NA}+15.2$ & 70 & 76 & 90 & 61 & 72 & 68 & 76 & 67 & 89 & 90 & 53 & 47 \\
\hline $\begin{array}{l}\text { Triadimenol-thiram+metalaxyl+ } \\
\text { thiram }\end{array}$ & $10.7+32.6+15.2+31.2$ & 77 & 78 & 88 & 70 & 76 & 71 & 63 & 68 & 79 & 86 & 47 & 40 \\
\hline Triadimenol+thiram+metalaxyl & $20.7+62.5+15.2$ & 74 & 79 & 86 & 62 & 65 & 62 & 68 & 71 & 89 & 85 & 52 & 51 \\
\hline \multirow[t]{2}{*}{$\begin{array}{l}\text { Chloroneb-TCMTB+myclobutanil+ } \\
\text { metalaxyl }\end{array}$} & $134.3+51.4+30.5+16.2$ & 68 & 76 & 84 & 60 & 65 & 66 & 74 & 66 & 84 & 89 & 53 & 46 \\
\hline & & & & & & & & & & \multicolumn{4}{|c|}{ (continued on next page) } \\
\hline
\end{tabular}

${ }^{\mathrm{t}}$ Numbers of trials conducted per year were: 17 in 1993, 18 in 1994, 17 in 1995, 20 in 1996, 18 in 1997,19 in 1998.

u A plus (+) between the fungicides indicates fungicides were separate formulations; a hyphen (-) between fungicides indicates one formulation.

${ }^{\mathrm{N}} \mathrm{NA}=$ a.i. rate not available.

${ }^{\mathrm{w}}$ TX1 = College Station: TX1a, 2 April planting date; TX1b, 27 April planting date.

${ }^{\mathrm{x}}$ Least significant difference to compare among treatments within a column and year for a trial with a significant $F$ test.

y Experimental products.

z Treatment not evaluated. 
Hora medium (30). Populations were corrected using a multipleinfection correction prior to calculating propagules per $100 \mathrm{~g}$ of soil. Soil populations of Pythium spp. and T. basicola were detected by diluting $25 \mathrm{~g}$ of soil, oven-dry weight equivalent, in $0.2 \%$ water agar to a total volume of $250 \mathrm{ml}$ and placing on a wrist action shaker for $20 \mathrm{~min}$. Pythium spp. were quantified by the spreadplate method on the selective medium $\mathrm{P}_{5} \mathrm{ARP}$, and T. basicola populations were quantified using the pour-plate method with the modified TB-CEN selective medium.
Statistics. Data were analyzed by the GLM procedure using SAS (SAS Institute Inc., Cary, NC). Percent stand was analyzed by location, because of a significant location by treatment interaction for all years, except 1999. Treatment means for trials having a significant $F$ test were separated by using a protected LSD at $P=$ 0.05 . For each trial with differences in percent stand, the difference between the percent stand for the seed treatment and nontreated seed was modeled nonparametrically using bivariate smoothing splines (35) as a function of minimal soil temperature and rainfall

Table 3. (continued from preceding page)

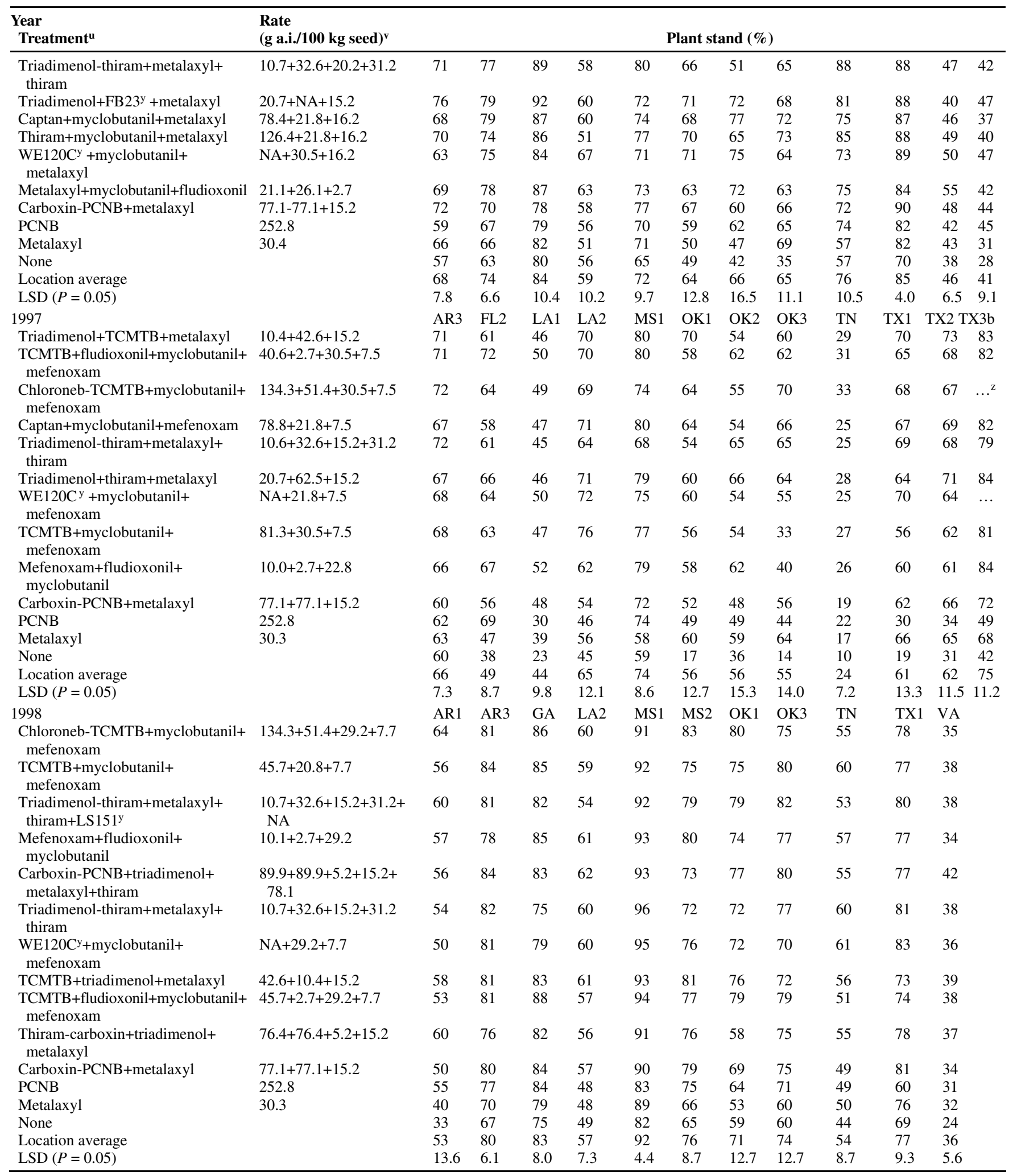


for the first 3 days after planting. Trials with less than a mean stand of $30 \%$ were eliminated for these models. These 12 of the 102 total trials with environmental data were considered as outliers and eliminated from the statistical analyses because stands were limited for agronomic reasons not related to the objectives of this study (e.g., soil crusting, heavy rainfall, or cold temperatures). When included, these results distorted the graphical presentation of the relationship between stand percentage, rainfall, and soil temperature after planting.

Mixed model analyses were used to compare stands for treatments. Product combinations used for comparisons included the fungicides azoxystrobin, myclobutanil, triadimenol, or PCNB each

Table 4. Plant stands for fungicide seed treatment combinations used for analyses from trials with a significant treatment response from the National Cottonseed Treatment Program for years with environmental data, 1999 to $2004^{\mathrm{v}}$

\begin{tabular}{|c|c|c|c|c|c|c|c|c|c|c|c|c|c|}
\hline $\begin{array}{l}\text { Year } \\
\text { Treatment }^{\mathrm{w}}\end{array}$ & $\begin{array}{l}\text { Rate } \\
(\text { g a.i./100 kg seed })^{x}\end{array}$ & & & & & & Plants & $\operatorname{tand}(c$ & & & & & \\
\hline 1999 & & AR3 & $\mathrm{CA}$ & LA2 & MS1 & OK2 & $\mathrm{TN}$ & TX1 & TX2 & TX3 & VA & & \\
\hline WE120C + myclobutanil+metalaxyl & $\mathrm{NA}+29.2+16.3$ & 72 & 75 & 70 & 70 & 82 & 59 & 79 & 35 & 72 & 45 & & \\
\hline Mefenoxam+fludioxonil+azoxystrobin & $7.7+2.7+4.9$ & 67 & 75 & 63 & 65 & 80 & 59 & 78 & 32 & 79 & 49 & & \\
\hline Chloroneb-TCMTB+myclobutanil+metalaxyl & $89.5+34.3+29.2+16.3$ & 64 & 76 & 82 & 64 & 81 & 47 & 78 & 32 & 78 & 48 & & \\
\hline Mefenoxam+fludioxonil+myclobutanil & $7.7+2.7+20.8$ & 73 & 77 & 72 & 63 & 78 & 57 & 80 & 34 & 72 & 40 & & \\
\hline Triadimenol+TCMTB+metalaxyl & $10.4+32.0+15.2$ & 72 & 72 & 72 & 67 & 84 & 56 & 79 & 46 & 74 & 44 & & \\
\hline Azoxystrobin+metalaxyl+TCMTB & $1.6+15.2+32.0$ & 68 & 75 & 76 & 68 & 74 & 45 & 81 & 38 & 75 & 50 & & \\
\hline Triadimenol-thiram+metalaxyl & $10.7+32.6+15.2$ & 74 & 73 & 82 & 70 & 80 & 56 & 80 & 38 & 73 & 42 & & \\
\hline TCMTB+myclobutanil+metalaxyl & $45.7+20.8+16.3$ & 66 & 77 & 78 & 62 & 75 & 50 & 78 & 51 & 78 & 43 & & \\
\hline Chloroneb+metalaxyl+myclobutanil+TCMTB & $270.9+15.2+20.8+45.7$ & 72 & 74 & 78 & 62 & 76 & 53 & 79 & 45 & 73 & 50 & & \\
\hline Carboxin-PCNB+metalaxyl+triadimenol & $77 \cdot 1+77.1+15.2+5.2$ & 68 & 75 & 69 & 65 & 82 & 54 & 81 & 37 & 73 & 49 & & \\
\hline Chloroneb+metalaxyl+myclobutanil & $270.9+15.2+20.8$ & 68 & 70 & 60 & 68 & 83 & 56 & 72 & 36 & 77 & 47 & & \\
\hline Mefenoxam+fludioxonil+myclobutanil & $7.7+2.7+29.2$ & 68 & 76 & 63 & 69 & 78 & 50 & 76 & 29 & 65 & 43 & & \\
\hline Carboxin-PCNB+metalaxyl & $77.1+77.1+15.2$ & 72 & 72 & 72 & 58 & 73 & 44 & 78 & 35 & 74 & 46 & & \\
\hline PCNB & 252.8 & 67 & 60 & 72 & 61 & 76 & 55 & 70 & 26 & 75 & 33 & & \\
\hline Metalaxyl & 30.5 & 62 & 68 & 67 & 52 & 73 & 38 & 73 & 27 & 68 & 34 & & \\
\hline None & & 56 & 66 & 62 & 56 & 65 & 37 & 68 & 23 & 70 & 30 & & \\
\hline Location average & & 68 & 72 & 72 & 64 & 78 & 51 & 77 & 35 & 73 & 44 & & \\
\hline $\operatorname{LSD}(P=0.05)^{\mathrm{z}}$ & & 7.6 & 6.2 & 13.3 & 9.6 & 9.2 & 13.8 & 7.8 & 11.1 & 8.0 & 7.7 & & \\
\hline 2000 & & $\mathrm{AL}$ & AR2 & AR3 & $\mathrm{CA}$ & LA1 & LA2 & MS1 & OK2 & OK3 & $\mathrm{TN}$ & TX1 & VA \\
\hline Triadimenol+TCMTB+metalaxyl & $10.4+32.0+15.2$ & 60 & 67 & 74 & 81 & 62 & 30 & 71 & 74 & 71 & 88 & 71 & 66 \\
\hline $\begin{array}{l}\text { Triadimenol-thiram+metalaxyl+TCMTB+ } \\
\text { Bacillus subtilis+carboxin-PCNB }\end{array}$ & $\begin{array}{l}10.7+32.6+20.3+32.0+ \\
0.4+89.9+89.9\end{array}$ & 63 & 79 & 68 & 72 & 66 & 28 & 72 & 78 & 72 & 86 & 74 & 66 \\
\hline Mefenoxam+fludioxonil+azoxystrobin & $7.7+2.7+19.7$ & 58 & 66 & 68 & 78 & 63 & 38 & 74 & 74 & 61 & 89 & 65 & 65 \\
\hline TCMTB+myclobutanil+mefenoxam & $45.7+19.7+24.1$ & 52 & 68 & 70 & 75 & 59 & 34 & 64 & 80 & 70 & 86 & 68 & 69 \\
\hline $\begin{array}{l}\text { Azoxystrobin-triadimenol-metalaxyl+ } \\
\text { TCMTB }\end{array}$ & $3.0+5.0+15.0+32.0$ & 53 & 74 & 73 & 76 & 60 & 28 & 71 & 66 & 64 & 88 & 66 & 70 \\
\hline $\begin{array}{l}\text { TCMTB+myclobutanil+mefenoxam+ } \\
\text { WE-143y + WE-144y }\end{array}$ & $\begin{array}{l}45.7+19.7+24.1 \\
\mathrm{NA}+\mathrm{NA}\end{array}$ & 60 & 75 & 69 & 76 & 55 & 36 & 70 & 75 & 72 & 86 & 64 & 63 \\
\hline Triadimenol-thiram+metalaxyl & $10.7+32.6+15.2$ & 58 & 70 & 73 & 78 & 56 & 29 & 67 & 73 & 65 & 85 & 74 & 69 \\
\hline Mefenoxam+fludioxonil+myclobutanil & $7.7+2.7+20.8$ & 46 & 71 & 68 & 72 & 60 & 34 & 67 & 69 & 56 & 86 & 70 & 66 \\
\hline Mefenoxam+fludioxonil+myclobutanil & $7.7+2.7+29.2$ & 54 & 70 & 71 & 71 & 58 & 30 & 68 & 74 & 40 & 88 & 71 & 71 \\
\hline $\begin{array}{l}\text { Chloroneb-TCMTB+myclobutanil+ } \\
\text { mefenoxam }\end{array}$ & $134.3+51.4+19.7+24.1$ & 55 & 70 & 60 & 71 & 62 & 24 & 71 & 72 & 61 & 87 & 66 & 62 \\
\hline Carboxin-PCNB+metalaxyl & $77.1+77.1+15.2$ & 45 & 65 & 66 & 75 & 52 & 25 & 66 & 72 & 61 & 84 & 65 & 58 \\
\hline PCNB & 252.8 & 49 & 58 & 63 & 74 & 44 & 15 & 65 & 73 & 53 & 82 & 57 & 60 \\
\hline Metalaxyl & 30.5 & 45 & 54 & 62 & 71 & 52 & 14 & 53 & 58 & 51 & 80 & 67 & 63 \\
\hline None & & 51 & 42 & 63 & 68 & 36 & 12 & 51 & 47 & 42 & 81 & 56 & 53 \\
\hline Location average & & 54 & 68 & 68 & 74 & 57 & 27 & 67 & 71 & 60 & 86 & 67 & 64 \\
\hline $\operatorname{LSD}(P=0.05)$ & & 11.5 & 8.3 & 8.2 & 7.1 & 9.8 & 14.0 & 8.8 & 12.5 & 15.8 & 3.5 & 9.6 & 9.3 \\
\hline 2001 & & AR3 & $\mathrm{CA}$ & GA & LA1 & LA2 & MS2 & $\mathrm{TN}$ & VA & & & & \\
\hline $\begin{array}{l}\text { Chloroneb-TCMTB+mefenoxam+ } \\
\text { myclobutanil }\end{array}$ & $134.4+51.4+24.1+21.0$ & 77 & 65 & 56 & 66 & 27 & 56 & 86 & 73 & & & & \\
\hline Triadimenol+TCMTB+metalaxyl & $10.4+32.0+15.2$ & 75 & 69 & 69 & 61 & 24 & 44 & 77 & 72 & & & & \\
\hline Triadimenol-thiram+metalaxyl & $10.7+33.0+15.2$ & 72 & 64 & 65 & 65 & 18 & 44 & 78 & 70 & & & & \\
\hline $\begin{array}{l}\text { Azoxystrobin+TCMTB+metalaxyl+ } \\
\text { triadimenol }\end{array}$ & $3.1+32.0+15.2+5.2$ & 76 & 61 & 62 & 59 & 8 & 56 & 82 & 65 & & & & \\
\hline Mefenoxam+WECO01B50+myclobutanil & $24.1+\mathrm{NA}+21.0$ & 72 & 66 & 62 & 59 & 20 & 44 & 81 & 66 & & & & \\
\hline Mefenoxam+fludioxonil+myclobutanil & $7.7+2.7+21.0$ & 76 & 61 & 68 & 60 & 18 & 47 & 83 & 67 & & & & \\
\hline $\begin{array}{l}\text { Triadimenol-thiram+metalaxyl+Bacillus } \\
\text { subtilis+carboxin-TCMTB-metalaxyl }\end{array}$ & $\begin{array}{l}10.7+33.0+15.2+0.5+ \\
91.0+190.3+15.2\end{array}$ & 74 & 63 & 38 & 57 & 30 & 52 & 78 & 70 & & & & \\
\hline Azoxystrobin+TCMTB+metalaxyl & $3.1+32.0+15.2$ & 72 & 47 & 58 & 64 & 13 & 43 & 81 & 71 & & & & \\
\hline Carboxin-PCNB+metalaxyl & $77.1+77.1+15.2$ & 70 & 50 & 64 & 65 & 11 & 56 & 78 & 68 & & & & \\
\hline PCNB & 252.8 & 61 & 38 & 61 & 12 & 6 & 30 & 72 & 40 & & & & \\
\hline Metalaxyl & 30.5 & 60 & 46 & 43 & 40 & 8 & 48 & 66 & 65 & & & & \\
\hline None & & 55 & 27 & 43 & 12 & 2 & 34 & 66 & 47 & & & & \\
\hline Location average & & 69 & 51 & 59 & 49 & 15 & 44 & 75 & 63 & & & & \\
\hline $\operatorname{LSD}(P=0.05)$ & & 8.3 & 9.2 & 15.9 & 13.3 & 12.7 & 11.6 & 7.3 & 8.7 & & & & \\
\hline
\end{tabular}

${ }^{v}$ Number of trials conducted per year were: 17 in 1999, 17 in 2000, 15 in 2001, 18 in 2002, 17 in 2003, and 18 in 2004

${ }^{w}$ A plus (+) between the fungicides indicates fungicides were separate formulations; a hyphen (-) between fungicides indicates one formulation.

${ }^{x} \mathrm{NA}=$ a.i. rate not available.

${ }^{\mathrm{y}}$ Experimental products.

${ }^{\mathrm{z}}$ Least significant difference to compare among treatments within a column and year for a trial with a significant $F$ test. 
with mefenoxam or metalaxyl. Pearson product-moment correlation was used to determine correlation coefficients among percent stand, disease, pathogen isolation frequency, and soil populations over locations. As a result of data for variables not being collected in some years or missing data, the number of observations for correlations is limited by the lowest number of observations for one of the variables $(n)$

\section{Results}

For the 211 replicated trials between 1993 and 2004 conducted by cooperators of the National Cottonseed Treatment Program, a significant $F$ test was found for 125 trials. However, a significant fungicide response was found for one or more treatments com- pared to no fungicide treatment in only 119 of these trials, or $56 \%$ of trials (Tables 3 to 5). Metalaxyl gave a significant stand response in 40 of the 119 trials. The selective activity of metalaxyl indicates the role of Pythium spp. in cotton stand establishment for these trials. A metalaxyl response was found at 18 of the 29 sites used in the evaluations, suggesting the widespread importance of this group of pathogens. PCNB gave a significant stand response in 44 of the 119 trials. The selectivity of PCNB, carboxin + PCNB in 1993, indicated a role for $R$. solani in stand establishment for these trials. The PCNB response was found for 19 of the 29 sites, suggesting the widespread importance of $R$. solani on cotton stand establishment. For PCNB and metalaxyl, a response for either fungicide was found in less than half of the evaluations, suggesting

Table 4. (continued from preceding page)

\begin{tabular}{|c|c|c|c|c|c|c|c|c|c|c|}
\hline \multirow{2}{*}{$\begin{array}{l}\text { Year } \\
\text { Treatment }^{\mathbf{w}} \\
2002\end{array}$} & \multirow[t]{2}{*}{$\begin{array}{l}\text { Rate } \\
(\mathrm{g} \text { a.i. } / 100 \mathrm{~kg} \text { seed })^{\mathrm{x}}\end{array}$} & \multicolumn{9}{|c|}{ Plant stand (\%) } \\
\hline & & AR1 & AR2 & AR3 & $\mathrm{CA}$ & GA & LA2 & OK2 & $\mathrm{TN}$ & VA \\
\hline $\begin{array}{l}\text { Azoxystrobin-fludioxonil-mefenoxam+ } \\
\text { myclobutanil }\end{array}$ & $14.6+2.4+7.3+21.0$ & 74 & 64 & 37 & 73 & 38 & 39 & 92 & 68 & 69 \\
\hline $\begin{array}{l}\text { Mefenoxam+chloroneb-TCMTB+ } \\
\text { myclobutanil }\end{array}$ & $24.1+134.3+51.4+25.0$ & 72 & 59 & 43 & 70 & 41 & 31 & 91 & 70 & 63 \\
\hline $\begin{array}{l}\text { Triadimenol-thiram+metalaxyl+azoxystrobin } \\
\text {-triadimenol-metalaxyl+Bacillus subtilis }\end{array}$ & $\begin{array}{l}10.7+32.6+15.2+ \\
3.0+5.0+15.0+0.5\end{array}$ & 72 & 54 & 53 & 72 & 36 & 30 & 89 & 69 & 65 \\
\hline Azoxystrobin-fludioxonil-mefenoxam & $14.6+2.4+7.3$ & 75 & 52 & 41 & 71 & 38 & 32 & 89 & 66 & 67 \\
\hline Azoxystrobin+TCMTB+metalaxyl & $3.0+21.3+15.2$ & 73 & 61 & 29 & 73 & 40 & 18 & 87 & 62 & 74 \\
\hline Triadimenol+TCMTB+metalaxyl & $10.4+21.3+15.2$ & 68 & 60 & 27 & 70 & 37 & 25 & 91 & 72 & 67 \\
\hline Azoxystrobin-fludioxonil-mefenoxam & $14.6+2.4+7.3$ & 69 & 51 & 43 & 68 & 35 & 30 & 90 & 67 & 70 \\
\hline Triadimenol-thiram+metalaxyl & $10.7+32.6+15.2$ & 66 & 55 & 46 & 66 & 35 & 32 & 90 & 71 & 66 \\
\hline Azoxystrobin-fludioxonil-mefenoxam & $14.6+2.4+7.3$ & 70 & 57 & 32 & 68 & 40 & 31 & 88 & 69 & 65 \\
\hline $\begin{array}{l}\text { Triadimenol-thiram+metalaxyl+Bacillus } \\
\text { subtilis+carboxin-TCMTB-metalaxyl }\end{array}$ & $\begin{array}{l}10.7+32.6+15.2+ \\
0.5+91.0+190.3+15.2\end{array}$ & 70 & 44 & 32 & 65 & 36 & 29 & 88 & 67 & 71 \\
\hline Carboxin-PCNB+metalaxyl & $77.1+77.1+15.2$ & 76 & 56 & 37 & 69 & 38 & 30 & 84 & 57 & 67 \\
\hline PCNB & 252.8 & 68 & 49 & 21 & 68 & 34 & 25 & 84 & 48 & 67 \\
\hline Metalaxyl & 30.5 & 60 & 46 & 23 & 57 & 15 & 14 & 82 & 39 & 56 \\
\hline None & $\ldots$ & 62 & 47 & 13 & 58 & 10 & 14 & 75 & 41 & 61 \\
\hline Location average & & 69 & 55 & 34 & 68 & 34 & 27 & 87 & 62 & 66 \\
\hline $\operatorname{LSD}(P=0.05)$ & & 8.6 & 9.1 & 13.0 & 6.0 & 11.9 & 12.2 & 5.1 & 8.0 & 6.4 \\
\hline 2003 & & AR1 & $\mathrm{CA}$ & GA & LA1 & LA2 & $\mathrm{TN}$ & TX2 & TX4 & \\
\hline $\begin{array}{l}\text { Mefenoxam+myclobutanil+chloroneb- } \\
\text { TCMTB }\end{array}$ & $24.1+43.5+259.7+99.5$ & 84 & 84 & 87 & 60 & 81 & 77 & 85 & 37 & \\
\hline $\begin{array}{l}\text { Azoxystrobin-fludioxonil-mefenoxam+ } \\
\text { myclobutanil }\end{array}$ & $14.7+2.5+7.4+21.0$ & 76 & 78 & 87 & 65 & 82 & 72 & 81 & 41 & \\
\hline Triadimenol+TCMTB+metalaxyl & $10.4+32.0+15.2$ & 79 & 80 & 84 & 50 & 83 & 82 & 83 & 39 & \\
\hline Azoxystrobin-fludioxonil-mefenoxam & $14.7+2.5+7.4$ & 72 & 75 & 86 & 64 & 80 & 74 & 87 & 41 & \\
\hline Mefenoxam+myclobutanil+TCMTB & $24.1+43.5+40.6$ & 73 & 82 & 86 & 67 & 85 & 71 & 79 & 25 & \\
\hline Triadimenol-thiram+metalaxyl & $10.7+32.6+15.2$ & 79 & 80 & 85 & 56 & 80 & 76 & 85 & 33 & \\
\hline $\begin{array}{l}\text { Mefenoxam+myclobutanil+TCMTB+ } \\
\text { WECO } 0257 \mathrm{y}\end{array}$ & $24.1+43.5+40.6+\mathrm{NA}$ & 78 & 80 & 85 & 52 & 82 & 74 & 82 & 39 & \\
\hline Azoxystrobin-fludioxonil-mefenoxam & $18.6+3.1+9.3$ & 79 & 80 & 85 & 49 & 74 & 75 & 79 & 40 & \\
\hline Metalaxyl+L0288 + triadimenol-thiram & $15.2+\mathrm{NA}+10.7+32.6$ & 79 & 79 & 83 & 68 & 78 & 80 & 81 & 34 & \\
\hline Carboxin-PCNB+metalaxyl & $77.1+77.1+15.2$ & 80 & 82 & 86 & 60 & 80 & 73 & 82 & 20 & \\
\hline PCNB & 252.8 & 66 & 69 & 88 & 66 & 77 & 53 & 79 & 12 & \\
\hline Metalaxyl & 30.5 & 66 & 67 & 75 & 36 & 74 & 68 & 78 & 26 & \\
\hline None & & 63 & 66 & 82 & 23 & 67 & 55 & 74 & 7 & \\
\hline Location average & & 75 & 77 & 84 & 54 & 79 & 70 & 81 & 28 & \\
\hline $\operatorname{LSD}(P=0.05)$ & & 10.0 & 11.1 & 7.0 & 15.7 & 9.0 & 7.8 & 6.4 & 13.2 & \\
\hline 2004 & & AR3 & $\mathrm{CA}$ & LA1 & OK3 & TX2 & TX4 & TX5 & & \\
\hline Azoxystrobin-fludioxonil-mefenoxam & $18.6+3.1+9.3$ & 70 & 73 & 74 & 82 & 63 & 91 & 64 & & \\
\hline $\begin{array}{l}\text { Azoxystrobin-fludioxonil-mefenoxam+ } \\
\text { myclobutanil }\end{array}$ & $14.7+2.5+7.4+21.0$ & 73 & 75 & 78 & 72 & 64 & 88 & 63 & & \\
\hline Triadimenol+TCMTB+metalaxyl & $10.4+32.0+15.2$ & 68 & 74 & 80 & 78 & 66 & 96 & 51 & & \\
\hline Azoxystrobin-fludioxonil-mefenoxam & $14.7+2.5+7.4$ & 64 & 73 & 79 & 68 & 60 & 86 & 49 & & \\
\hline $\mathrm{L} 1226^{\mathrm{y}}+$ metalaxyl+triadimenol & $\mathrm{NA}+20.3+6.6$ & 68 & 72 & 80 & 76 & 63 & 93 & 50 & & \\
\hline Metalaxyl+L0288 + triadimenol-thiram & $15.2+\mathrm{NA}+10.7+32.6$ & 66 & 75 & 81 & 78 & 57 & 91 & 59 & & \\
\hline $\begin{array}{l}\text { Mefenoxam+WECO } 0257^{y}+\text { myclobutanil+ } \\
\text { chloroneb-TCMTB }\end{array}$ & $\begin{array}{l}23.4+\mathrm{NA}+43.5+134.3 \\
+51.4\end{array}$ & 71 & 76 & 79 & 78 & 60 & 90 & 44 & & \\
\hline Triadimenol-thiram+metalaxyl & $10.7+32.6+15.2$ & 67 & 75 & 77 & 80 & 61 & 89 & 47 & & \\
\hline Mefenoxam+WECO $0257^{\mathrm{y}}+$ myclobutanil & $23.4+\mathrm{NA}+43.5$ & 73 & 73 & 77 & 78 & 61 & 92 & 38 & & \\
\hline Carboxin-PCNB+metalaxyl & $77.1+77.1+15.2$ & 62 & 70 & 75 & 73 & 59 & 88 & 57 & & \\
\hline PCNB & 252.8 & 64 & 69 & 66 & 68 & 57 & 90 & 48 & & \\
\hline Metalaxyl & 30.5 & 62 & 69 & 60 & 57 & 50 & 82 & 49 & & \\
\hline None & & 60 & 64 & 44 & 65 & 48 & 80 & 43 & & \\
\hline Location average & & 66 & 72 & 72 & 74 & 59 & 89 & 47 & & \\
\hline $\operatorname{LSD}(P=0.05)$ & & 6.7 & 4.1 & 9.5 & 14.7 & 8.6 & 6.1 & 20.4 & & \\
\hline
\end{tabular}


a role for other pathogens or a complex of pathogens in disease development. Carboxin + PCNB + metalaxyl, the historic standard fungicide treatment, improved stands for 78 of the 119 trials where a response was observed compared to no fungicide treatment (Tables 3 to 5). In $38 \%$ of the trials where a response was observed (45 trials), one or more of the nominated fungicide combinations increased stand compared to the historic standard fungicide treatment. In all years except 1999, the best fungicide combination or combinations improved stand for all trials or all but one trial.
From the nominations across years, selected treatments were used to compare specific changes in chemistries used as seed treatments by the industry. These new chemistries, triazoles (myclobutanil or triadimenol) and azoxystrobin, were compared to carboxin + PCNB, with all products being used in combination with either metalaxyl or mefenoxam. For 390 observations, the mean stands for azoxystrobin, $63.1 \%$, or triazoles, $63.0 \%$, were greater than the historic combination, carboxin + PCNB, $61.2 \%(P=0.0036)$. Comparison of these same chemistries for trials (95 observations) with $20 \%$ or more isolation frequency of $T$. basicola found stands of $65.8,64.2$, and

Table 5. Ratio of significant responses over seed without fungicides to total number of trials for a site ${ }^{\mathrm{v}}$ (significant response over the historic standard ${ }^{\mathrm{w}}$ ) for fungicide seed treatment combinations used for analyses from trials for the National Cottonseed Treatment Program, 1993 to $2004^{\mathrm{x}}$

\begin{tabular}{|c|c|c|c|c|c|c|c|c|}
\hline \multicolumn{9}{|l|}{ Seed treatment } \\
\hline & $\mathrm{AL}$ & AR1 & AR2 & AR3 & $\mathrm{CA}$ & FL1 & FL2 & GA \\
\hline Metalaxyl+thiram+triadimenol & $2 / 19(1)$ & $9 / 15(1)$ & $8 / 19(0)$ & $13 / 17(4)$ & $6 / 16(2)$ & $1 / 11(0)$ & $2 / 2(1)$ & $5 / 19(4)$ \\
\hline Metalaxyl+myclobutanil+captan & $1 / 4(0)$ & $3 / 4(0)$ & $2 / 4(0)$ & $1 / 3(0)$ & $0 / 4(0)$ & $0 / 3(0)$ & & $0 / 4(0)$ \\
\hline Metalaxyl+myclobutanil+TCMTB & $1 / 5(0)$ & $4 / 5(1)$ & $3 / 5(0)$ & $2 / 4(0)$ & $1 / 5(0)$ & $0 / 3(0)$ & & $0 / 5(3)$ \\
\hline Metalaxyl+myclobutanil & $0 / 1(0)$ & $1 / 1(0)$ & $0 / 1(0)$ & & $0 / 1(0)$ & & & $0 / 1(0)$ \\
\hline Chloroneb+metalaxyl+myclobutanil ${ }^{\mathrm{y}}$ & $0 / 3(0)$ & $2 / 3(0)$ & $1 / 3(0)$ & $1 / 2(0)$ & $0 / 3(0)$ & $0 / 1(0)$ & & $0 / 3(0)$ \\
\hline Metalaxyl+TCMTB+triadimenol & $0 / 9(1)$ & $3 / 6(0)$ & $3 / 9(0)$ & $8 / 9(2)$ & $5 / 7(1)$ & $0 / 3(0)$ & $1 / 1(0)$ & $2 / 9(1)$ \\
\hline Metalaxyl+myclobutanil+thiram & $0 / 3(0)$ & $2 / 3(1)$ & $3 / 3(0)$ & $3 / 3(0)$ & $0 / 3(0)$ & $0 / 3(0)$ & & $1 / 3(2)$ \\
\hline Chloroneb+metalaxyl+myclobutanil+TCMTB & $0 / 4(0)$ & $1 / 4(0)$ & $2 / 4(0)$ & $4 / 4(0)$ & $2 / 4(0)$ & $0 / 2(0)$ & & $0 / 4(0)$ \\
\hline Metalaxyl+PCNB+thiram+triadimenol & $0 / 1(0)$ & $1 / 1(0)$ & $1 / 1(0)$ & $0 / 1(0)$ & $0 / 1(0)$ & $0 / 1(0)$ & & $1 / 1(1)$ \\
\hline Metalaxyl+myclobutanil+exp. ${ }^{\mathrm{z}}$ & $0 / 2(0)$ & $0 / 2(0)$ & $0 / 2(0)$ & $2 / 2(0)$ & $1 / 2(0)$ & $1 / 1(0)$ & & $0 / 2(0)$ \\
\hline Fludioxonil+metalaxyl+myclobutanil & $0 / 1(0)$ & $0 / 1(0)$ & $1 / 1(0)$ & $1 / 1(0)$ & $0 / 1(0)$ & $0 / 1(0)$ & & $0 / 1(0)$ \\
\hline Metalaxyl+triadimenol+exp. ${ }^{\mathrm{z}}$ & $0 / 3(0)$ & $0 / 3(0)$ & $2 / 3(0)$ & $3 / 3(0)$ & $2 / 3(0)$ & $0 / 2(0)$ & & $0 / 3(0)$ \\
\hline Fludioxonil+mefenoxam+myclobutanil+TCMTB & $0 / 2(0)$ & $1 / 1(0)$ & $0 / 2(0)$ & $2 / 2(1)$ & & $0 / 2(0)$ & $1 / 1(1)$ & $1 / 2(1)$ \\
\hline Chloroneb+mefenoxam+myclobutanil+TCMTB & $0 / 6(0)$ & $3 / 3(1)$ & $2 / 6(0)$ & $4 / 6(1)$ & $3 / 4(1)$ & $0 / 2(0)$ & $1 / 1(0)$ & $2 / 6(0)$ \\
\hline Captan+myclobutanil+mefenoxam & $0 / 1(0)$ & & $0 / 1(0)$ & $0 / 1(0)$ & & $0 / 1(0)$ & $1 / 1(0)$ & $0 / 1(0)$ \\
\hline Mefenoxam+myclobutanil+exp. ${ }^{\mathrm{z}}$ & $0 / 4(0)$ & $1 / 2(0)$ & $0 / 4(0)$ & $4 / 4(2)$ & $2 / 2(1)$ & $0 / 2(0)$ & $1 / 1(0)$ & $1 / 4(0)$ \\
\hline Mefenoxam+myclobutanil+TCMTB & $0 / 4(0)$ & $2 / 2(0)$ & $1 / 4(0)$ & $2 / 4(1)$ & $1 / 2(0)$ & $0 / 2(0)$ & $1 / 1(0)$ & $1 / 4(0)$ \\
\hline Fludioxonil+mefenoxam+myclobutanil & $0 / 7(0)$ & $1 / 3(0)$ & $2 / 7(0)$ & $4 / 7(0)$ & $3 / 5(0)$ & $0 / 2(0)$ & $1 / 1(1)$ & $2 / 7(0)$ \\
\hline Metalaxyl+thiram+triadimenol+exp. ${ }^{\mathrm{z}}$ & $0 / 3(0)$ & $2 / 3(0)$ & $0 / 3(0)$ & $1 / 3(0)$ & $2 / 2(1)$ & $0 / 1(0)$ & & $0 / 3(0)$ \\
\hline Carboxin+metalaxyl+PCNB+thiram+triadimenol & $0 / 1(0)$ & $1 / 1(0)$ & $0 / 1(0)$ & $1 / 1(0)$ & & $0 / 1(0)$ & & $0 / 1(0)$ \\
\hline Carboxin+metalaxyl+thiram+triadimenol & $0 / 1(0)$ & $1 / 1(0)$ & $0 / 1(0)$ & $1 / 1(0)$ & & $0 / 1(0)$ & & $0 / 1(0)$ \\
\hline Carboxin+metalaxyl+PCNB+triadimenol & $0 / 1(0)$ & $0 / 1(0)$ & $0 / 1(0)$ & $1 / 1(0)$ & $1 / 1(0)$ & & & $0 / 1(0)$ \\
\hline Azoxystrobin+fludioxonil+mefenoxam & $0 / 9(1)$ & $2 / 8(0)$ & $2 / 9(0)$ & $5 / 9(0)$ & $8 / 9(0)$ & & & $3 / 9(0)$ \\
\hline Azoxystrobin+metalaxyl+TCMTB & $0 / 3(0)$ & $1 / 2(0)$ & $1 / 3(0)$ & $3 / 3(0)$ & $3 / 3(0)$ & & & $1 / 3(0)$ \\
\hline $\begin{array}{l}\text { Carboxin+metalaxyl+PCNB+TCMTB+thiram+triadimenol+ } \\
\text { Bacillus subtilis }\end{array}$ & $1 / 1(1)$ & & $1 / 1(1)$ & $0 / 1(0)$ & $0 / 1(0)$ & & & $0 / 1(0)$ \\
\hline Mefenoxam+myclobutanil+TCMTB+exp. ${ }^{\mathrm{z}}$ & $0 / 2(1)$ & $1 / 1(0)$ & $1 / 2(1)$ & $0 / 2(0)$ & $2 / 2(0)$ & & & $0 / 2(0)$ \\
\hline Azoxystrobin+metalaxyl+TCMTB+triadimenol & $0 / 2(1)$ & & $1 / 2(1)$ & $2 / 2(0)$ & $2 / 2(1)$ & & & $1 / 2(0)$ \\
\hline $\begin{array}{l}\text { Carboxin+metalaxyl+TCMTB+thiram+triadimenol+ } \\
\text { Bacillus subtilis }\end{array}$ & $0 / 2(0)$ & $0 / 1(0)$ & $0 / 2(0)$ & $2 / 2(0)$ & $2 / 2(1)$ & & & $1 / 2(0)$ \\
\hline Azoxystrobin+fludioxonil-mefenoxam+myclobutanil & $0 / 3(0)$ & $2 / 3(0)$ & $1 / 3(0)$ & $2 / 3(1)$ & $3 / 3(1)$ & & & $1 / 3(0)$ \\
\hline $\begin{array}{l}\text { Azoxystrobin+metalaxyl+thiram+triadimenol+ } \\
\text { Bacillus subtilis }\end{array}$ & $0 / 1(0)$ & $1 / 1(0)$ & $0 / 1(0)$ & $1 / 1(0)$ & $1 / 1(0)$ & & & $1 / 1(0)$ \\
\hline Chloroneb+mefenoxam + myclobutanil+TCMTB + exp. ${ }^{\mathrm{z}}$ & $0 / 1(0)$ & $0 / 1(0)$ & $0 / 1(0)$ & $1 / 1(1)$ & $1 / 1(1)$ & & & $0 / 1(0)$ \\
\hline Carboxin-PCNB+metalaxyl & $1 / 12$ & $6 / 9$ & $3 / 12$ & $5 / 11$ & $4 / 10$ & $0 / 5$ & $1 / 1$ & $4 / 12$ \\
\hline PCNB & $0 / 12(0)$ & $3 / 9(0)$ & $1 / 12(0)$ & $3 / 11(0)$ & $3 / 10(0)$ & $0 / 5(0)$ & $1 / 1(1)$ & $3 / 12(0)$ \\
\hline \multirow[t]{2}{*}{ Metalaxyl } & $1 / 12(0)$ & $2 / 9(0)$ & $4 / 12(0)$ & $0 / 11(0)$ & $2 / 10(0)$ & $0 / 5(0)$ & $1 / 1(0)$ & $0 / 12(0)$ \\
\hline & LA1 & LA2 & MS1 & MS2 & OK1 & $\mathrm{OK} 2$ & OK3 & $\mathrm{SC}$ \\
\hline Metalaxyl+thiram+triadimenol & $14 / 19(0)$ & $11 / 19(1)$ & $7 / 19(2)$ & $2 / 19(1)$ & $8 / 18(0)$ & $8 / 14(3)$ & $11 / 17(0)$ & $0 / 7(0)$ \\
\hline Metalaxyl+myclobutanil+captan & $3 / 4(0)$ & $3 / 4(0)$ & $1 / 4(0)$ & $2 / 4(0)$ & 2/4(1) & $1 / 4(0)$ & $1 / 4(0)$ & $0 / 3(0)$ \\
\hline Metalaxyl+myclobutanil+TCMTB & $3 / 5(0)$ & $1 / 5(0)$ & $1 / 5(0)$ & $1 / 5(1)$ & $4 / 4(0)$ & $1 / 2(0)$ & $3 / 5(0)$ & $0 / 4(0)$ \\
\hline Metalaxyl+myclobutanil & $0 / 1(0)$ & $0 / 1(0)$ & $0 / 1(0)$ & $1 / 1(1)$ & $1 / 1(0)$ & $0 / 1(0)$ & $0 / 1(0)$ & $0 / 1(0)$ \\
\hline Chloroneb+metalaxyl+myclobutanil & $1 / 3(0)$ & $1 / 3(0)$ & $1 / 3(1)$ & $1 / 3(1)$ & $1 / 2(0)$ & $1 / 3(1)$ & $0 / 3(0)$ & $0 / 2(0)$ \\
\hline Metalaxyl+TCMTB+triadimenol & $6 / 9(1)$ & $5 / 9(2)$ & $4 / 9(0)$ & $1 / 9(0)$ & $3 / 8(1)$ & $4 / 6(2)$ & $3 / 7(0)$ & $0 / 1(0)$ \\
\hline Metalaxyl+myclobutanil+thiram & $3 / 3(0)$ & $0 / 3(0)$ & $0 / 3(0)$ & $0 / 3(0)$ & $3 / 3(0)$ & $1 / 1(0)$ & $3 / 3(0)$ & $0 / 2(0)$ \\
\hline Chloroneb+metalaxyl+myclobutanil+TCMTB & $2 / 4(0)$ & $2 / 4(0)$ & $0 / 4(0)$ & $0 / 4(0)$ & $2 / 2(0)$ & $3 / 3(0)$ & $2 / 4(0)$ & $0 / 1(0)$ \\
\hline Metalaxyl+PCNB+thiram+triadimenol & $1 / 1(0)$ & $0 / 1(0)$ & $0 / 1(0)$ & $0 / 1(0)$ & $1 / 1(0)$ & & $1 / 1(0)$ & $0 / 1(0)$ \\
\hline Metalaxyl+myclobutanil+exp. ${ }^{\mathrm{z}}$ & $1 / 2(0)$ & $0 / 2(0)$ & $1 / 2(1)$ & $0 / 2(0)$ & $1 / 1(0)$ & $2 / 2(0)$ & $1 / 2(0)$ & \\
\hline Fludioxonil+metalaxyl+myclobutanil & $1 / 1(0)$ & $0 / 1(0)$ & $0 / 1(0)$ & $0 / 1(0)$ & $1 / 1(0)$ & $1 / 1(0)$ & $1 / 1(0)$ & \\
\hline Metalaxyl+triadimenol+exp. ${ }^{\mathrm{z}}$ & $2 / 3(0)$ & $0 / 3(0)$ & $0 / 3(0)$ & $0 / 3(0)$ & $2 / 3(0)$ & $2 / 3(0)$ & $2 / 3(0)$ & \\
\hline Fludioxonil+mefenoxam+myclobutanil+TCMTB & $1 / 3(0)$ & $2 / 2(1)$ & $2 / 2(0)$ & $1 / 2(0)$ & $2 / 2(0)$ & $1 / 2(0)$ & $2 / 2(0)$ & \\
\hline Chloroneb+mefenoxam+myclobutanil+TCMTB & $4 / 6(1)$ & $5 / 6(2)$ & $3 / 6(0)$ & $2 / 6(0)$ & $2 / 6(0)$ & $3 / 4(1)$ & $3 / 4(0)$ & \\
\hline Captan+myclobutanil+mefenoxam & $1 / 1(0)$ & $1 / 1(1)$ & $1 / 1(0)$ & $0 / 1(0)$ & $1 / 1(0)$ & $1 / 1(0)$ & $1 / 1(0)$ & \\
\hline Mefenoxam+myclobutanil+exp. ${ }^{\mathrm{z}}$ & $3 / 5(0)$ & $3 / 4(1)$ & $2 / 4(1)$ & $1 / 4(0)$ & $2 / 4(0)$ & $1 / 3(0)$ & $1 / 3(0)$ & \\
\hline
\end{tabular}

\footnotetext{
v Fisher's protected least significant difference, $P=0.05$

${ }^{\mathrm{w}}$ Historic fungicide standard carboxin+PCNB+metalaxyl.

${ }^{x}$ Fungicide combinations are listed in the chronological order (year) they first appeared.

y In 1994, fungicide combination included Trichoderma harzianum.

$\mathrm{z}$ Treatments included one or more experimental products.
} 
$62.1 \%$ for triazoles (triadimenol or myclobutanil), azoxystrobin, and carboxin + PCNB, respectively. For these trials, triazoles were greater than carboxin $+\mathrm{PCNB}$, but azoxystrobin was not significantly different from either chemistry $(P=0.0009)$.

Correlations were examined for the association of disease symptoms, isolation frequency, and soil populations of selected pathogen groups to plant stand. Percent stand was negatively correlated with the hypocotyl $(r=-0.28, P<0.0001, n=204)$ and root disease indices $(r=-0.20, P=0.0038, n=204)$, respectively. Mean plant stands for trials also were negatively correlated with soil populations of Pythium spp. $(r=-0.32, P<0.0001, n=179)$, with a weak association with isolation of Pythium spp. on $\mathrm{P}_{5}$ ARP $(r=$ $-0.23, P=0.0617, n=67)$ and $R$. solani $(r=-0.12, P=0.0843, n$
$=202$ ), respectively. The hypocotyl disease index was positively correlated with isolation frequency of $R$. solani $(r=0.37, P<$ $0.0001, n=201)$ and T. basicola, $(r=0.28, P<0.0001, n=202)$. The root disease index was positively correlated with isolation frequency of $T$. basicola $(r=0.26, P=0.0002, n=202)$. In addition, isolation frequency was positively correlated with soil populations for $R$. solani $(r=0.32, P<0.0001, n=183)$ and $T$. basicola $(r=0.54, P<0.0001, n=183)$. Isolation frequency of Pythium was associated with soil population counts of Pythium on water agar $(r=0.14, P=0.06, n=176)$ or $\mathrm{P}_{5} \mathrm{ARP}(r=0.41, P=0.0009$, $n=64)$. Isolation of Fusarium spp. was negatively correlated with isolation of $R$. solani $(r=-0.34, P<0.0001, n=202)$ and $T$. basicola $(r=-0.26, P=0.0002, n=202)$.

Table 5. (continued from preceding page)

\begin{tabular}{|c|c|c|c|c|c|c|c|c|}
\hline \multicolumn{9}{|l|}{ Seed treatment } \\
\hline Mefenoxam+myclobutanil+TCMTB & $3 / 5(0)$ & $4 / 4(1)$ & $3 / 4(0)$ & $1 / 4(0)$ & $2 / 4(0)$ & $2 / 3(0)$ & $3 / 3(0)$ & \\
\hline Fludioxonil+mefenoxam+myclobutanil & $4 / 8(0)$ & $5 / 7(0)$ & $5 / 7(1)$ & $2 / 7(0)$ & $2 / 5(0)$ & $5 / 6(0)$ & $2 / 6(0)$ & \\
\hline Metalaxyl+thiram+triadimenol+exp. ${ }^{\mathrm{z}}$ & $2 / 4(0)$ & $1 / 3(0)$ & $1 / 3(0)$ & $1 / 3(0)$ & $1 / 3(0)$ & $0 / 2(0)$ & $1 / 2(0)$ & \\
\hline Carboxin+metalaxyl+PCNB+thiram+triadimenol & $0 / 2(0)$ & $1 / 1(0)$ & $1 / 1(0)$ & $0 / 1(0)$ & $1 / 1(0)$ & $0 / 1(0)$ & $1 / 1(0)$ & \\
\hline Carboxin+metalaxyl+thiram+triadimenol & $0 / 2(0)$ & $0 / 1(0)$ & $1 / 1(0)$ & $1 / 1(0)$ & $0 / 1(0)$ & $0 / 1(0)$ & $1 / 1(0)$ & \\
\hline Carboxin+metalaxyl+PCNB+triadimenol & $0 / 1(0)$ & $0 / 1(0)$ & $0 / 1(0)$ & $0 / 1(0)$ & & $1 / 1(0)$ & $0 / 1(0)$ & \\
\hline Azoxystrobin+fludioxonil+mefenoxam & $5 / 6(1)$ & $5 / 9(0)$ & $1 / 9(0)$ & $0 / 9(0)$ & $0 / 8(0)$ & $5 / 7(1)$ & $2 / 7(0)$ & \\
\hline Azoxystrobin+metalaxyl+TCMTB & $1 / 2(0)$ & $1 / 3(0)$ & $1 / 3(1)$ & $0 / 3(0)$ & $0 / 2(0)$ & $1 / 2(0)$ & $0 / 2(0)$ & \\
\hline $\begin{array}{l}\text { Carboxin+metalaxyl+PCNB+TCMTB+thiram+triadimenol+ } \\
\text { Bacillus subtilis }\end{array}$ & $1 / 1(1)$ & $1 / 1(0)$ & $1 / 1(0)$ & $0 / 1(0)$ & $0 / 1(0)$ & $1 / 1(0)$ & $1 / 1(0)$ & \\
\hline Mefenoxam+myclobutanil+TCMTB+exp. ${ }^{z}$ & $2 / 2(0)$ & $2 / 2(0)$ & $1 / 2(0)$ & $0 / 2(0)$ & $0 / 2(0)$ & $1 / 1(0)$ & $1 / 1(0)$ & \\
\hline Azoxystrobin+metalaxyl+TCMTB+triadimenol & $2 / 2(0)$ & $1 / 2(0)$ & $1 / 2(0)$ & $1 / 2(0)$ & $0 / 2(0)$ & $1 / 1(0)$ & $1 / 1(0)$ & \\
\hline $\begin{array}{l}\text { Carboxin+metalaxyl+TCMTB+thiram+triadimenol+ } \\
\text { Bacillus subtilis }\end{array}$ & $1 / 1(0)$ & $2 / 2(0)$ & $0 / 2(0)$ & $1 / 2(0)$ & $0 / 2(0)$ & $1 / 1(0)$ & $0 / 1(0)$ & \\
\hline Azoxystrobin+fludioxonil-mefenoxam+myclobutanil & $2 / 2(0)$ & $2 / 3(0)$ & $0 / 3(0)$ & $0 / 3(0)$ & $0 / 3(0)$ & $1 / 2(1)$ & $0 / 2(0)$ & \\
\hline $\begin{array}{l}\text { Azoxystrobin+metalaxyl+thiram+triadimenol+ } \\
\text { Bacillus subtilis }\end{array}$ & & $1 / 1(0)$ & $0 / 1(0)$ & $0 / 1(0)$ & $0 / 1(0)$ & $1 / 1(0)$ & $0 / 1(0)$ & \\
\hline Chloroneb+mefenoxam+myclobutanil+TCMTB+exp. ${ }^{z}$ & $1 / 1(0)$ & $0 / 1(0)$ & $0 / 1(0)$ & $0 / 1(0)$ & & & & \\
\hline Carboxin-PCNB+metalaxyl & $8 / 12$ & $3 / 12$ & $3 / 12$ & $2 / 12$ & $3 / 11$ & $3 / 9$ & $5 / 10$ & $0 / 3$ \\
\hline $\mathrm{PCNB}$ & $3 / 12(0)$ & $1 / 12(0)$ & $2 / 12(0)$ & $3 / 12(1)$ & $3 / 11(0)$ & $4 / 9(0)$ & $3 / 10(0)$ & $0 / 3(0)$ \\
\hline \multirow[t]{2}{*}{ Metalaxyl } & $4 / 12(0)$ & $1 / 12(0)$ & $1 / 12(0)$ & $1 / 12(0)$ & $1 / 11(0)$ & $3 / 9(0)$ & $2 / 10(0)$ & $0 / 3(0)$ \\
\hline & $\mathrm{TN}$ & TX1 & TX2 & TX3 & TX4 & TX5 & VA & \\
\hline Metalaxyl+thiram+triadimenol & $16 / 19(5)$ & $17 / 18(1)$ & $13 / 19(0)$ & $2 / 18(1)$ & $2 / 3(0)$ & $0 / 3(0)$ & $4 / 7(1)$ & \\
\hline Metalaxyl+myclobutanil+captan & $4 / 4(0)$ & $5 / 5(1)$ & $1 / 4(0)$ & $0 / 2(0)$ & & & & \\
\hline Metalaxyl+myclobutanil+TCMTB & $4 / 5(0)$ & $6 / 6(0)$ & $4 / 5(1)$ & $0 / 4(0)$ & & & $1 / 1(0)$ & \\
\hline Metalaxyl+myclobutanil & $1 / 1(0)$ & $2 / 2(0)$ & $0 / 1(0)$ & & & & & \\
\hline Chloroneb+metalaxyl+myclobutanil & $3 / 3(0)$ & $3 / 4(0)$ & $1 / 3(0)$ & $0 / 1(0)$ & & & $1 / 1(0)$ & \\
\hline Metalaxyl+TCMTB+triadimenol & $8 / 9(3)$ & $4 / 6(0)$ & $5 / 9(0)$ & $1 / 9(0)$ & $2 / 3(2)$ & $0 / 3(0)$ & $4 / 7(0)$ & \\
\hline Metalaxyl+myclobutanil+thiram & $3 / 3(0)$ & $3 / 3(0)$ & $3 / 3(0)$ & $0 / 4(0)$ & & & & \\
\hline Chloroneb+metalaxyl+myclobutanil+TCMTB & $3 / 4(0)$ & $4 / 4(0)$ & $3 / 4(0)$ & $0 / 5(0)$ & & & $2 / 2(0)$ & \\
\hline Metalaxyl+PCNB+thiram+triadimenol & $1 / 1(0)$ & $1 / 1(0)$ & $1 / 1(0)$ & $0 / 1(0)$ & & & & \\
\hline Metalaxyl+myclobutanil+exp..$^{z}$ & $2 / 2(1)$ & $2 / 2(0)$ & $2 / 2(0)$ & $0 / 390)$ & & & $1 / 1(0)$ & \\
\hline Fludioxonil+metalaxyl+myclobutanil & $1 / 1(0)$ & $1 / 1(0)$ & $1 / 1(0)$ & $0 / 2(0)$ & & & & \\
\hline Metalaxyl+triadimenol+ exp..$^{\mathrm{z}}$ & $2 / 3(0)$ & $1 / 2(0)$ & $3 / 3(0)$ & $0 / 4(0)$ & $1 / 1(0)$ & $0 / 1(0)$ & $0 / 1(0)$ & \\
\hline Fludioxonil+mefenoxam+myclobutanil+TCMTB & $1 / 2(1)$ & $1 / 2(0)$ & $1 / 2(0)$ & $1 / 3(0)$ & & & $1 / 1(0)$ & \\
\hline Chloroneb+mefenoxam+myclobutanil+TCMTB & $6 / 6(3)$ & $2 / 4(0)$ & $2 / 6(0)$ & $0 / 7(0)$ & $1 / 2(1)$ & $0 / 2(0)$ & $2 / 5(0)$ & \\
\hline Captan+myclobutanil+mefenoxam & $1 / 1(0)$ & $1 / 1(0)$ & $1 / 1(0)$ & $1 / 2(0)$ & & & & \\
\hline Mefenoxam+myclobutanil+exp. ${ }^{\mathrm{z}}$ & $3 / 4(1)$ & $2 / 3(0)$ & $2 / 4(0)$ & $0 / 3(0)$ & $1 / 1(0)$ & $0 / 1(0)$ & $2 / 3(0)$ & \\
\hline Mefenoxam+myclobutanil+TCMTB & $4 / 4(2)$ & $2 / 3(0)$ & $1 / 4(0)$ & $1 / 5(0)$ & $1 / 1(0)$ & $0 / 1(0)$ & $2 / 3(1)$ & \\
\hline Fludioxonil+mefenoxam+myclobutanil & $6 / 7(1)$ & $5 / 7(0)$ & $1 / 7(0)$ & $1 / 8(0)$ & & & $6 / 6(1)$ & \\
\hline Metalaxyl+thiram+triadimenol+exp. ${ }^{\mathrm{z}}$ & $2 / 3(0)$ & $1 / 1(0)$ & $2 / 3(0)$ & $0 / 2(0)$ & $2 / 2(1)$ & $0 / 2(0)$ & $1 / 3(0)$ & \\
\hline Carboxin+metalaxyl+PCNB+thiram+triadimenol & $1 / 1(0)$ & $0 / 1(0)$ & $0 / 1(0)$ & $0 / 1(0)$ & & & $1 / 1(1)$ & \\
\hline Carboxin+metalaxyl+thiram+triadimenol & $1 / 1(0)$ & $0 / 1(0)$ & $0 / 1(0)$ & $0 / 1(0)$ & & & $1 / 1(0)$ & \\
\hline Carboxin+metalaxyl+PCNB+triadimenol & $1 / 1(0)$ & $1 / 1(0)$ & $1 / 1(0)$ & $0 / 1(0)$ & & & $1 / 1(0)$ & \\
\hline Azoxystrobin+fludioxonil+mefenoxam & $7 / 9(5)$ & $1 / 2(0)$ & $3 / 9(0)$ & $1 / 7(0)$ & $3 / 7(2)$ & $1 / 7(0)$ & $4 / 9(0)$ & \\
\hline Azoxystrobin+metalaxyl+TCMTB & $2 / 3(0)$ & $1 / 2(0)$ & $1 / 3(0)$ & $0 / 3(0)$ & $0 / 1(0)$ & $0 / 1(0)$ & $3 / 3(1)$ & \\
\hline $\begin{array}{l}\text { Carboxin+metalaxyl+PCNB+TCMTB+thiram+triadimenol+ } \\
\text { Bacillus subtilis }\end{array}$ & $1 / 1(0)$ & $1 / 1(0)$ & $0 / 1(0)$ & $0 / 1(0)$ & & & $1 / 1(0)$ & \\
\hline Mefenoxam+myclobutanil+TCMTB+exp. ${ }^{z}$ & $2 / 2(0)$ & $0 / 1(0)$ & $1 / 2(0)$ & $0 / 2(0)$ & $1 / 1(1)$ & $0 / 1(0)$ & $1 / 2(0)$ & \\
\hline Azoxystrobin+metalaxyl+TCMTB+triadimenol & $2 / 2(1)$ & $1 / 2(0)$ & $0 / 2(0)$ & $0 / 2(0)$ & & & $2 / 2(1)$ & \\
\hline $\begin{array}{l}\text { Carboxin+metalaxyl+TCMTB+thiram+triadimenol+ } \\
\text { Bacillus subtilis }\end{array}$ & $2 / 2(1)$ & $0 / 1(0)$ & $0 / 2(0)$ & $0 / 2(0)$ & $0 / 1(0)$ & $0 / 1(0)$ & $2 / 2(0)$ & \\
\hline Azoxystrobin+fludioxonil-mefenoxam+myclobutanil & $2 / 3(1)$ & & $2 / 3(0)$ & $0 / 2(0)$ & $2 / 3(1)$ & $0 / 3(0)$ & $1 / 3(0)$ & \\
\hline $\begin{array}{l}\text { Azoxystrobin+metalaxyl+thiram+triadimenol+ } \\
\text { Bacillus subtilis }\end{array}$ & $1 / 1(1)$ & & $0 / 1(0)$ & $0 / 1(0)$ & $0 / 1(0)$ & $0 / 1(0)$ & $0 / 1(0)$ & \\
\hline Chloroneb+mefenoxam+myclobutanil+TCMTB + exp. $^{\mathrm{z}}$ & $0 / 1(0)$ & & $1 / 1(0)$ & & $1 / 1(0)$ & $0 / 1(0)$ & $0 / 1(0)$ & \\
\hline Carboxin+PCNB+metalaxyl & $8 / 12$ & $8 / 10$ & $6 / 12$ & $1 / 11$ & $1 / 3$ & $0 / 3$ & $3 / 7$ & \\
\hline PCNB & $4 / 12(0)$ & $2 / 10(0)$ & $2 / 12(0)$ & $0 / 11 /(0)$ & $1 / 3(0)$ & $0 / 3(00$ & $2 / 7(0)$ & \\
\hline Metalaxyl & 4/12(0) & $6 / 10(0)$ & $2 / 12(0)$ & 1/11(1) & $1 / 3(0)$ & $0 / 3(0)$ & $3 / 7(0)$ & \\
\hline
\end{tabular}


Environment had a large impact on stand establishment, with stand being similar at the highest minimal soil temperatures of $25^{\circ} \mathrm{C}$ across rainfall amounts of no rain to $62 \mathrm{~mm}$ the first 3 days after planting (Fig. 1). Mean stand over plotted trials was 55\%. As soil temperature the first 3 days after planting decreased among trials to the minimal soil temperature examined of $12^{\circ} \mathrm{C}$, stands decreased. In addition, an increase in stand reduction at these lower temperatures was seen as rainfall increased (Fig. 1). The role of environment on seedling disease severity was examined as a change in stand between the standard carboxin + PCNB + metalaxyl treatment and seed not receiving a fungicide treatment for trials (Fig. 2A). The mean stand improvement for carboxin + PCNB + metalaxyl was 10.4. Under all environmental conditions, fungicides improved stand compared to seed not receiving fungicides (Fig. 2A). Lowest stand improvements occurred at a minimal soil temperature of $25^{\circ} \mathrm{C}$. As rainfall amounts increased and soil temperatures decreased for trials, seed treatment fungicides increased stands more compared to nontreated seed. The greatest seedling disease pressure or stand increases with fungicides occurred for rainfall at lower soil temperatures compared to higher soil temperatures for the carboxin + PCNB + metalaxyl treatment (Fig. 2A). An additional profile was developed for nominations containing a triazole fungicide and metalaxyl or mefenoxam and nontreated seed to observe the response of newer

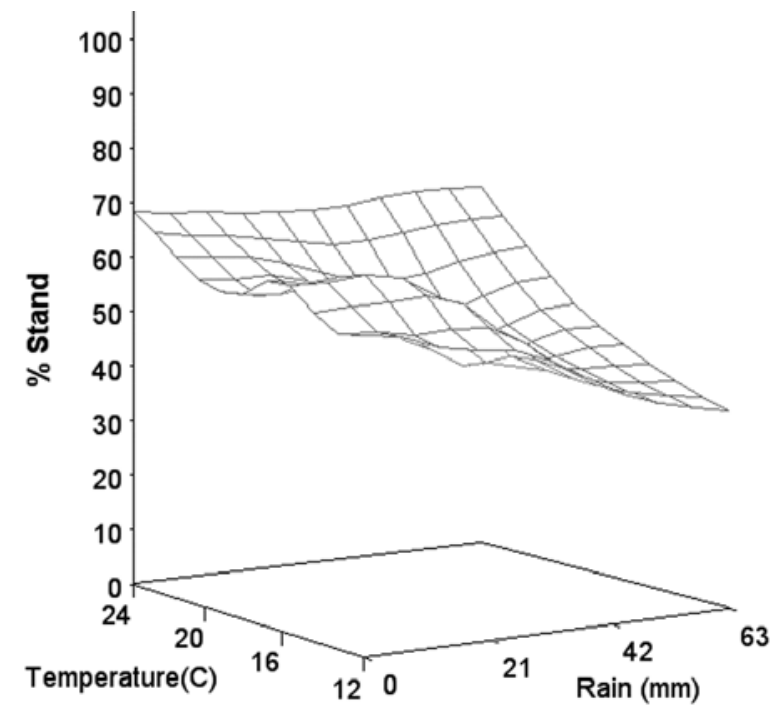

Fig. 1. Percent stand for the mean of trials from 1999 to 2004 in relation to rainfall and average minimal soil temperature for the first 3 days after planting. chemistries across environments (Fig. 2B). The mean stand improvement for a triazole and metalaxyl or mefenoxam was 13.2. A very similar profile was observed for stand difference over environments as for carboxin + PCNB + metalaxyl. However, increases in stand with these fungicide products at reduced minimal soil temperatures at planting and increasing rainfall were greater than observed with the historic standard fungicide.

Environment also influenced the importance of pathogen groups in the seedling disease complex. Plotting the stand difference between the metalaxyl seed treatment and no fungicide treatment over environment showed that metalaxyl gave a mean improvement of 5.9 (Fig. 3). Little change in stand response was found for metalaxyl over the minimal soil temperatures of 12 to $25^{\circ} \mathrm{C}$ in the absence of rainfall the first 3 days after planting. However, as rainfall after planting increased at lower minimal soil temperatures, a metalaxyl response was evident, with stand differences of close to 20 at $62 \mathrm{~mm}$ of rainfall and a minimal soil temperature of $12^{\circ} \mathrm{C}$. For stand differences between stands for the PCNB seed treatment compared to no fungicide seed treatment, a mean stand change of $3.6 \%$ was found over environments (Fig. 4). Few differences were evident over the range of temperatures of 12 to $25^{\circ} \mathrm{C}$ and rainfall amounts of none to $62 \mathrm{~mm}$, although there was a suggestion of greater response with the combination of low soil temperature and high rainfall amounts.

\section{Discussion}

Value of fungicide seed treatments. The National Cottonseed Treatment Trials from 1993 to 2004 documented the importance of seedling diseases in stand establishment problems. Of 212 replicated trials conducted over 12 years and 29 locations, seed treatment fungicides significantly improved stand in 119 trials over a range of soil types and planting environments. This work supports the widespread use of fungicide seed treatments on cotton. It is common to use multiple fungicides to target the range of seedling disease pathogens. Davis et al. (12) over a 3-year period in the San Joaquin Valley observed a response to the fungicides myclobutanil + metalaxyl or myclobutanil in 22 of 25 trials. Metalaxyl alone improved stands only in trials in 1995, but not the 18 trials in 1993 or 1994 . Kaufman et al. (28) demonstrated the value of seed treatments in improving cotton stands in Texas. This study also demonstrated reduced root necrosis and yield increases from use of triazole fungicides at a site with a history of black root rot (28). Wheeler et al. (65) also demonstrated yield increases from seed treatment fungicides.

Historically, carboxin + PCNB + metalaxyl were used widely to limit stand reductions from $R$. solani and Pythium spp. The availability of new or alternative chemistries for the control of seedling diseases is important as older chemistries, such as PCNB, are not being reregistered for use on cotton. In these studies, newer
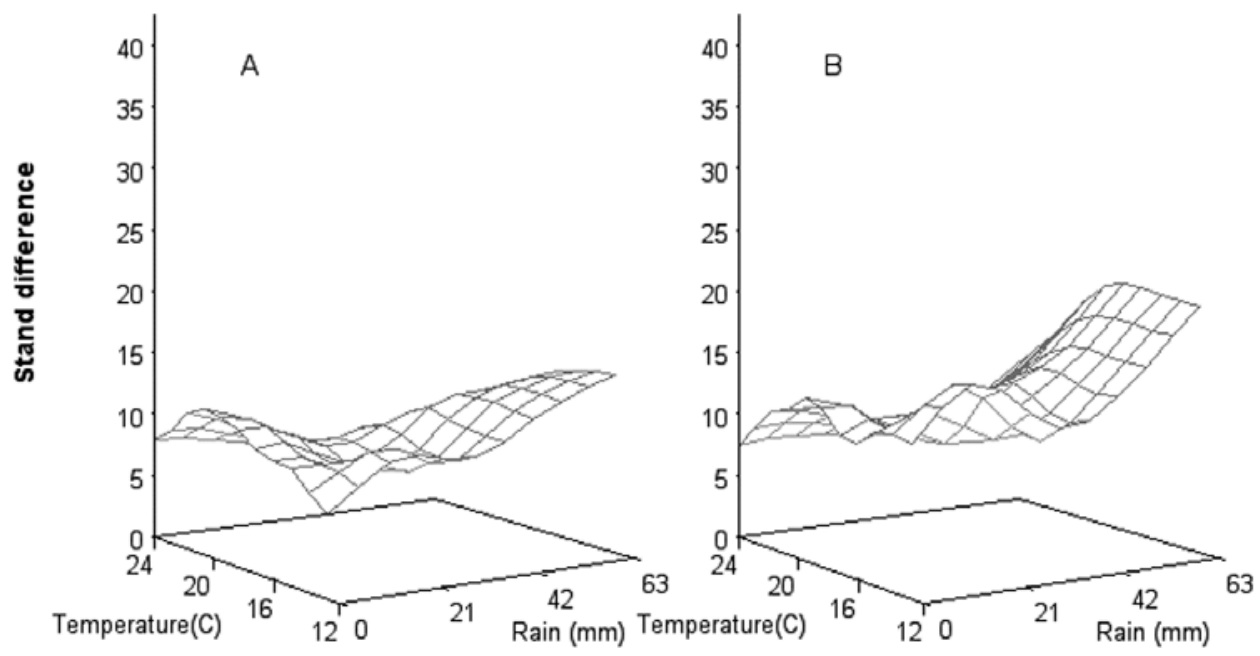

Fig. 2. Difference in mean stand percentages between seed treated with A, carboxin + PCNB + metalaxyl or B, a triazole and either metalaxyl or mefenoxam and seed with no fungicide treatment for trials from 1999 to 2004 in relation to rainfall and average minimal soil temperature for the first 3 days after planting. 
chemistries for the control of $R$. solani and other pathogens, azoxystrobin and triazole fungicides, were shown to significantly improve stands when used in combination with mefenoxam or metalaxyl compared to carboxin + PCNB + metalaxyl. Fichtner et al. (16) also observed that triadimenol with thiram and metalaxyl was more effective in reducing root necrosis and hypocotyl discoloration compared to seed treatment with carboxin + PCNB + mefenoxam. Newer chemistries such as the triazoles are also increasing the spectrum of seedling diseases being controlled, with the triazoles providing some control of $T$. basicola $(28,63)$. This was evident at the sites for these trials having black root rot, with stands for seed treatments containing triazole fungicides being more effective that other fungicide chemistries.

Role of environment. Stand reductions were evident over sites as soil temperatures decreased and rainfall increased the first 3 days after planting. Poor stands and an increase in seedling diseases are often associated with early planting $(10,26)$. Johnson et al. (26) found poor stands at soil temperatures of $10^{\circ} \mathrm{C}$ or lower, with good stands at minimal soil temperatures of $19^{\circ} \mathrm{C}$ or higher. In Louisiana, Colyer et al. (10) found plant populations were low in an early April planting, increased in late April and early May plantings, and remained high for a mid-May planting. Planting too early is not recommended, as it is often associated with low temperatures and abundant rainfall that increase abiotic stresses, including soil crusting, soil anoxia, and chilling injury, and increase susceptibility to seedling disease pathogens. However, research has not clearly defined the role of environmental or soil factors on the relative importance of seedling diseases associated with reduced stands for cotton. In these studies, fungicide response compared to seed not receiving a fungicide treatment clearly identified the role of seedling diseases in stand differences across environments. Fungicide stand response increased as stands decreased with decreasing soil temperature and increasing soil water content. Incidence and severity of cotton seedling diseases have been shown previously to be affected by soil temperature and moisture content $(6,26,34)$. However, in these studies stand responses were not associated with chilling injury that may increase seedling diseases caused by $R$. solani or Pythium spp., since the soil responses were examined at minimal soil temperatures of $12^{\circ} \mathrm{C}$ and above $(7,31,34,61)$. Davis et al. (12) found good fungicide response over environments with mean soil temperatures that ranged from 19.7 to $22.2^{\circ} \mathrm{C}$ for the first 5 days after planting, suggesting that even at favorable soil temperatures seedling diseases can be important in stand establishment.

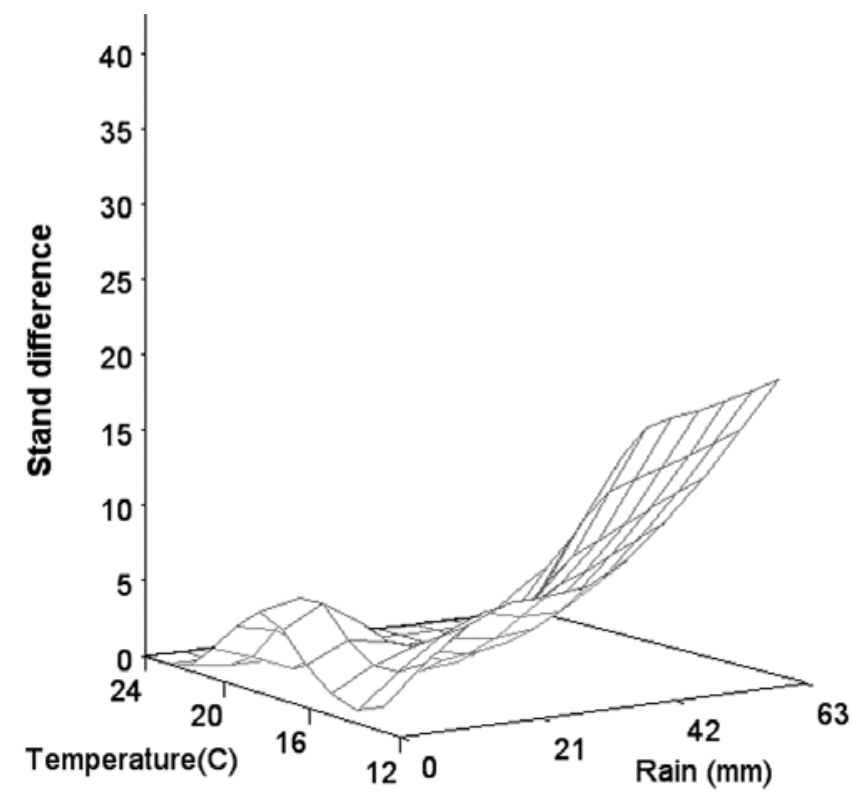

Fig. 3. Difference in mean stand percentages between seed with a metalaxyl fungicide treatment and no fungicide treatment for trials from 1999 to 2004 in relation to rainfall and average minimal soil temperature for the first 3 days after planting.
The use of fungicide treatments with selective spectrums of activity also indicated the importance of stand losses associated with seedling diseases caused by Pythium spp. or R. solani. Stand losses from Pythium spp. clearly increased as soil temperature decreased from a minimal soil temperature of 25 to $12^{\circ} \mathrm{C}$ and with increasing rainfall the first 3 days after planting. This supports earlier research from Tennessee that found in 7 years of field studies, soil moisture and temperature at planting directly affected isolation frequency of Pythium, with isolation being negatively correlated with minimal soil temperature and positively correlated with soil moisture content (26). These observations on the role of soil temperature and moisture on the severity of seedling disease caused by different Pythium spp. is supported by controlled studies. Optimal temperature for root rot in cotton caused by $P$. irregulare was $15^{\circ} \mathrm{C}$, over a range of temperatures from 15 to $31^{\circ} \mathrm{C}$ (43). Arndt (3) found P. ultimum caused the most damage at 18 and $21^{\circ} \mathrm{C}$ over a temperature range of 18 to $30^{\circ} \mathrm{C}$. Black root rot of cotton caused by $T$. basicola is also more severe under cool wet environments after planting $(5,32,44)$.

Response from the PCNB seed treatment compared to no fungicide treatment varied little with soil environment after planting, suggesting stand losses from $R$. solani were not influenced greatly by soil temperature or rainfall the first 3 days after planting. Previous controlled temperature studies by Arndt (4) found $R$. solani to be most destructive at 18 to $33^{\circ} \mathrm{C}$. Similarly, Hunter et al. (22) found activity from 20 to $32^{\circ} \mathrm{C}$ for three isolates of $R$. solani. Walker (64) found stand losses from $R$. solani over a similar range of temperatures and also found that the pathogen reduced stand over a wide range of soil water contents. Based on isolation frequency from field studies, seedlings were equally susceptible when grown at minimal soil temperatures ranging from 14 to $24^{\circ} \mathrm{C}$, and susceptibility was not associated with soil moisture (26).

Relation of pathogen isolation and population to stand establishment. Limited research has tried to examine the importance of soil pathogen populations on disease development. In these trials, a significant negative correlation was found between Pythium soil populations and stand, but not other pathogens examined. Isolation frequency was positively correlated with soil populations of $R$. solani, Pythium spp., and T. basicola. Soil population explained only a small proportion of the variability in stands observed across sites and years. DeVay et al. (14) observed that survival of cotton seedlings from nontreated seeds was negatively correlated with populations of $P$. ultimum in field soils. Other studies in California

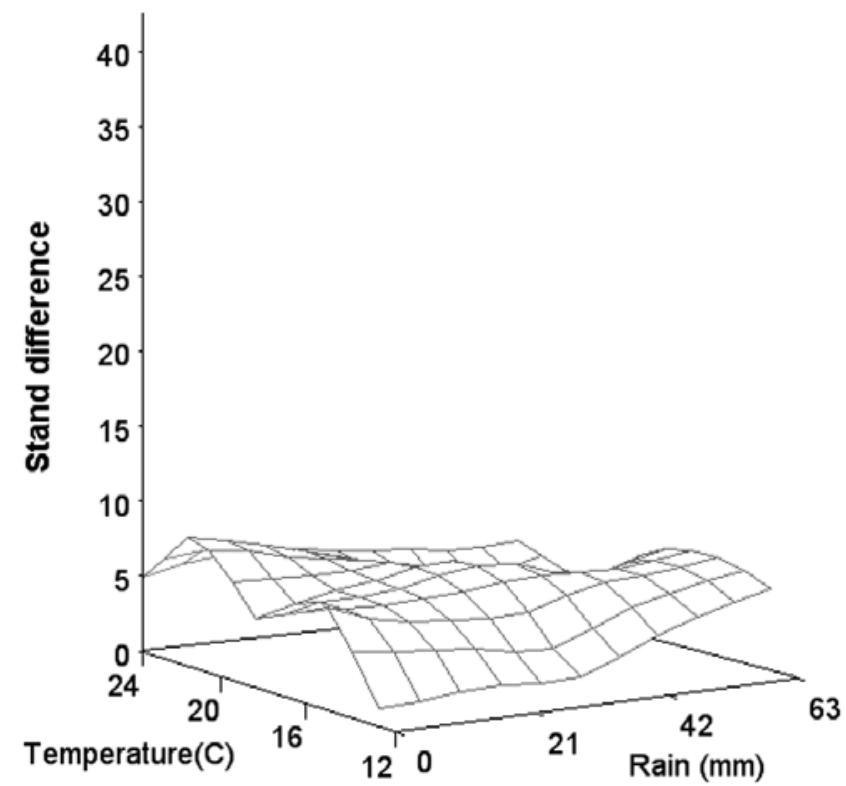

Fig. 4. Difference in mean stand percentages between seed with a PCNB fungicide treatment and no fungicide treatment for trials from 1999 to 2004 in relation to rainfall and average minimal soil temperature for the first three days after planting. 
found stands decreased as populations of $R$. solani and Pythium increased for 12 trials, and the decrease was related to response of fungicide with activity to specific pathogen groups (18). However, Davis et al. (12) did not find an association between $R$. solani or Pythium soil populations and stands. Johnson and Doyle (27) in Tennessee also found no relationship between Pythium soil populations and isolation frequency or disease on cotton seedlings. This information along with the environmental response of fungicides suggests soil pathogen populations are not the primary factor in determining seedling disease severity on cotton.

These multi-year data support the widespread use of seed treatment fungicides for cotton. Newer seed treatment fungicides with QoI or triazole chemistries were demonstrated to be superior to older fungicide seed treatment chemistries. Although pathogen populations were shown to be associated with stand establishment or disease severity, environment appeared to be more important in determining stand establishment across sites and years.

\section{Acknowledgments}

We thank the participation and financial support of Bayer CropScience, Syngenta, Wilbur-Ellis, Helena Chemical Company, Buckman Laboratories, and ISK Biotech Corp.

\section{Literature Cited}

1. Abd-Elsalam, K. A., Asran-Amal, A-M., Omar, M. R., and Aly, A. A. 2006. Frequency and diversity of Fusarium spp. colonizing roots of Egyptian cottons. Arch. Phytopathol. Plant Prot. 39:165-177.

2. Allen, S. J. 2001. Black root rot. Pages 16-17 in: Compendium of Cotton Diseases. T. L. Kirkpatrick and C. S. Rothrock, eds. American Phytopathological Society, St. Paul, MN.

3. Arndt, C. H. 1943. Pythium ultimum and the damping-off of cotton seedlings. Phytopathology 33:607-611.

4. Arndt, C. H. 1957. Temperature as a factor in the infection of cotton seedlings by ten pathogens. Plant Dis. Rep. Suppl. 246:63-84.

5. Blank, L. M., Leyendecker, P. J., Jr., and Nakayama, R. M. 1953. Observations on black root rot symptoms on cotton seedlings at different soil temperatures. Plant Dis. Rep. 37:473-476.

6. Brown, E. A., and McCarter, S. M. 1976. Effect of a seedling disease caused by Rhizoctonia solani on subsequent growth and yield of cotton. Phytopathology 66:111-115.

7. Christiansen, M. N. 1964. Influence of chilling upon subsequent growth and morphology of cotton seedlings. Crop Sci. 4:584-586.

8. Colyer, P. D. 1988. Frequency and pathogenicity of Fusarium spp. associated with seedling diseases of cotton in Louisiana. Plant Dis. 72:400-402.

9. Colyer, P. D. 2001. Diseases caused by Fusarium species. Pages 18-19 in: Compendium of Cotton Diseases. T. L. Kirkpatrick and C. S. Rothrock, eds. American Phytopathological Society, St. Paul, MN.

10. Colyer, P. D., Micinski, S., and Nguyen, K. T. 1991. Effect of planting date on the efficiency of an in-furrow pesticide and the development of cotton seedling disease. Plant Dis. 75:739-742.

11. Davis, R. G. 1975. Microorganisms associated with diseased cotton seedlings in Mississippi. Plant Dis. Rep. 59:277-280.

12. Davis, R. M., Nunez, J. J., and Subbarao, K. V. 1997. Benefits of cotton seed treatments for the control of seedling diseases in relation to inoculum densities of Pythium species and Rhizoctonia solani. Plant Dis. 81:766-768.

13. DeVay, J. E. 2001. Seedling Diseases. Pages 13-14 in: Compendium of Cotton Diseases. T. L. Kirkpatrick and C. S. Rothrock, eds. American Phytopathological Society, St. Paul, MN.

14. DeVay, J. E., Garber, R. H., and Matheron, D. 1982. Role of Pythium species in the seedling disease complex of cotton in California. Plant Dis. 66:151-154.

15. DeVay, J. E., and Rothrock, C. S. 2001. Control of seedling diseases. Pages 19-20 in: Compendium of Cotton Diseases. T. L. Kirkpatrick and C. S. Rothrock, eds. American Phytopathological Society, St. Paul, MN.

16. Fichtner, S. M., Isakeit, T., Wheeler, T. A., Kaufman, H. W., and Gannaway, J. R. 2005. Evaluation of several approaches to manage Meloidogyne incognita and cotton seedling disease complexes in the high plains of Texas. J. Nematol. 37:66-70.

17. Fulton, N. D., and Bollenbacher, K. 1959. Pathogenicity of fungi isolated from diseased cotton seedlings. Phytopathology 49:684-689.

18. Garber, R. H., DeVay, J. E., Weinhold, A. R., and Matheron, D. 1979. Relationship of pathogen inoculum to cotton seedling disease control with fungicides. Plant Dis. Rep. 63:246-250.

19. Henis, Y., Ghaffar, A., Baker, R., and Gillespie, S. L. 1978. A new pellet soil-sampler and its use for the study of population dynamics of Rhizoctonia solani in soil. Phytopathology 68:371-376.

20. Hillocks, R. J. 1992. Seedling diseases. Pages 1-38 in: Cotton Diseases. R. J. Hillocks, ed. CAB Intl., Wallingford, UK.

21. Howell, C. R. 2001. Diseases caused by Pythium species. Pages 14-15 in: Compendium of Cotton Diseases. T. L. Kirkpatrick and C. S. Rothrock, eds. American Phytopathological Society, St. Paul, MN.
22. Hunter, R. E., Staffeldt, E. E., and Maier, C. R. 1960. Effects of soil temperature on the pathogenicity of Rhizoctonia solani isolates. Plant Dis. Rep. 44:793-795.

23. Jeffers, S. N., and Martin, S. B. 1986. Comparison of two media selective for Phytophthora and Pythium species. Plant Dis. 70:1038-1040.

24. Johnson, L. F., Baird, D. D., Chambers, A. Y., and Shamiyeh, N. B. 1978. Fung associated with postemergence seedling disease of cotton in three soils. Phytopathology 68:917-920.

25. Johnson, L. F., and Chambers, A. Y. 1973. Isolation and identity of three species of Pythium that cause cotton seedling blight. Plant Dis. Rep. 57:848-852.

26. Johnson, L. F., Chambers, A. Y., and Measells, J. W. 1969. Influence of soil moisture, temperature, and planting date on severity of cotton seedling blight. Tennessee Agric. Exp. Stn. Bull. 461.

27. Johnson, L. F., and Doyle, J. H. 1986. Relationship of seedling disease of cotton to characteristics of loessial soils in Tennessee. Phytopathology 76:286-290.

28. Kaufman, H., Wheeler, T., Graves, R., Schuster, G., Kidd, P., and Siders, K. 1998. Large plot performance of seedling disease seed treatment fungicides. Vol. 1, pages 149-152 in: Proc. Beltwide Cotton Prod. Conf. National Cotton Council of America, Memphis, TN.

29. Kerby, T. A., Keeley, M., and Johnson, S. 1989. Weather and seed quality variables to predict cotton seedling emergence. Agron. J. 81:415-419.

30. Ko, W., and Hora, F. K. 1971. A selective medium for the quantitative determination of Rhizoctonia solani in soil. Phytopathology 61:707-710.

31. Ludwig, C. A. 1932. The germination of cottonseed at low temperatures. J. Agric. Res. 44:367-380.

32. Maier, C. R. 1966. Implication in chemical control presented by the variability of cotton seedling disease pathogens. Plant Dis. Rep. 50:301305.

33. Mathre, D. E., Ravenscroft, A. V., and Garber, R. H. 1966. The role of Thielaviopsis basicola as a primary cause of yield reduction in cotton in California. Phytopathology 56:1213-1216.

34. McCarter, S. M., and Roncadori, R. W. 1971. Influence of low temperature during cottonseed germination on growth and disease susceptibility. Phytopathology 61:1426-1429.

35. Meinguet, J. 1979. Multivariate interpolation at arbitrary points made simple. J. Appl. Math. Physics 30:292-304.

36. Melero-Vara, J. M., and Jimenaz-Diaz, R. M. 1990. Etiology, incidence, and distribution of cotton seedling damping-off in southern Spain. Plant Dis. 74:597-600.

37. Minton, E. B., and Garber, R. H. 1983. Controlling the seedling disease complex of cotton. Plant Dis. 67:115-118.

38. Ogle, H. J., Stirling, A. M., and Dart, P. J. 1993. Pathogenicity of fungi associated with seedling disease of cotton. Aust. J. Exp. Agric. 33:923-929.

39. Palmateer, A. J., McLean, K. S., Morgan-Jones, G., and van Santen, E. 2004. Frequency and diversity of fungi colonizing tissues of upland cotton. Mycopathologia 157:303-316.

40. Ranney, C. D. 1962. Fungi involved in the seedling disease complex of cotton in the Yazoo-Mississippi Delta. Plant Dis. Rep. 46:122-123.

41. Ray, W. W., and McLaughlin, J. H. 1942. Isolation and infection tests with seedand soil-borne cotton pathogens. Phytopathology 32:233-238.

42. Riley, J. A., Newton, O. H., Measells, J. W., Downey, D. A., and Hand, L. 1964. Soil temperatures and cotton planting in the mid-South. Miss. Agric. Exp. Stn. Bull. 678 .

43. Roncadori, R. W., and McCarter, S. W. 1972. Effect of soil treatment, soil temperature, and plant age on Pythium root rot of cotton. Phytopathology 62:373-376.

44. Rothrock, C. S. 1992. Influence of soil temperature, water, and texture on Thielaviopsis basicola and black root rot of cotton. Phytopathology 82:1202-1206.

45. Rothrock, C. S. 1994. Report of the cottonseed treatment committee for 1993. Pages 216-218 in: Proc. Beltwide Cotton Prod. Conf. National Cotton Council of America, Memphis, TN.

46. Rothrock, C. S. 1996. Report of the cottonseed treatment committee for 1995. Vol. 1, pages 234-238 in: Proc. Beltwide Cotton Prod. Conf. National Cotton Council of America, Memphis, TN.

47. Rothrock, C. S. 1996. Cotton diseases incited by Rhizoctonia solani. Pages 269-277 in: Rhizoctonia Species: Taxonomy, Molecular Biology, Ecology, Pathology and Disease Control. B. Sneh, S. Jabaji-Hare, S. Neate, and G. Dijst, eds. Kluwer Academic Publishers, Boston.

48. Rothrock, C. S. 1997. Report of the cottonseed treatment committee for 1996. Vol. 1, pages 126-131 in: Proc. Beltwide Cotton Prod. Conf. National Cotton Council of America, Memphis, TN

49. Rothrock, C. S. 1997. Prevalence and distribution of Thielaviopsis basicola Pages 75-76 in: Proc. Beltwide Cotton Prod. Conf. National Cotton Council of America, Memphis, TN.

50. Rothrock, C. S. 1998. Report of the cottonseed treatment committee for 1997. Vol. 1, pages 107-111 in: Proc. Beltwide Cotton Prod. Conf. National Cotton Council of America, Memphis, TN.

51. Rothrock, C. S. 1999. Report of the cottonseed treatment committee for 1998. Vol. 1, pages 92-96 in: Proc. Beltwide Cotton Prod. Conf. National Cotton Council of America, Memphis, TN

52. Rothrock, C. S. 2001. Report of the cottonseed treatment committee - 2000. Vol. 1, pages 89-93 in: Proc. Beltwide Cotton Prod. Conf. National Cotton Council of America, Memphis, TN. 
53. Rothrock, C. S., and Miller, P. K. 1995. Report of the cottonseed treatment committee for 1994. Pages 185-189 in: Proc. Beltwide Cotton Prod. Conf. National Cotton Council of America, Memphis, TN.

54. Rothrock, C. S., and Winters, S. A. 2000. Report of the cottonseed treatment committee for 1999. Vol. 1, pages 119-123 in: Proc. Beltwide Cotton Prod. Conf. National Cotton Council of America, Memphis, TN.

55. Rothrock, C. S., and Winters, S. A. 2002. Report of the cottonseed treatment committee for 2001. In: Proc. Beltwide Cotton Prod. Conf. National Cotton Council of America, Memphis, TN.

56. Rothrock, C. S., and Winters, S. A. 2003. Report of the cottonseed treatment committee for 2002. Pages A19-A24 in: Proc. Beltwide Cotton Prod. Conf. National Cotton Council of America, Memphis, TN.

57. Rothrock, C. S., and Winters, S. A. 2004. Report of the cottonseed treatment committee for 2003. Pages 452-458 in: Proc. Beltwide Cotton Prod. Conf. National Cotton Council of America, Memphis, TN.

58. Rothrock, C. S., and Winters, S. A. 2005. Report of the cottonseed treatment committee for 2004. Pages 249-256 in: Proc. Beltwide Cotton Prod. Conf. National Cotton Council of America, Memphis, TN.

59. Roy, K. W., and Bourland, F. M. 1982. Epidemiological and mycofloral relationships in cotton seedling disease in Mississippi. Phytopathology 72:868-872.
60. Rude, P. A. 1984. Integrated Pest Management for Cotton in the Western Region of the United States. University of California, Division of Agriculture and Natural Resources Pub. 3305.

61. Shao, F. M., and Christianson, M. N. 1982. Cotton seedling radicle exudates in relation to susceptibility to Verticillium wilt and Rhizoctonia root rot Phytopathol. Z. 105:351-359.

62. Specht, L. P., and Griffin, G. J. 1985. A selective medium for enumerating low populations of Thielaviopsis basicola. Can. J. Plant Pathol. 7:438-441.

63. Toksoz, H., Rothrock, C. S., and Kirkpatrick, T. L. 2009. Efficacy of seed treatment chemicals for black root rot, caused by Thielaviopsis basicola, on cotton. Plant Dis. 93:354-362.

64. Walker, M. N. 1928. Soil temperature studies with cotton. III. Relation of soil temperature and soil moisture to the soreshin disease of cotton. Univ. Florida Agric. Exp. Stn. Bull. 197.

65. Wheeler, T. A., Gannaway, J. R., Kaufman, H. W., Dever, J. K., Mertley, J. C., and Keeling, J. W. 1997. Influence of tillage, seed quality, and fungicide seed treatments on cotton emergence and yield. J. Prod. Agric 10:394-400.

66. Wheeler, T. A., Hake, K. D., and Dever, J. K. 2000. Survey of Meloidogyne incognita and Thielaviopsis basicola: Their impact on cotton fruiting and producers' management choices in infested fields. J. Nematol. 32(4S):576-583. 